\title{
Liquid biopsies come of age: towards implementation of circulating tumour DNA
}

Jonathan C. M. Wan ${ }^{1,2}$, Charles Massie ${ }^{1,2}$, Javier Garcia-Corbacho ${ }^{3}$, Florent Mouliere $^{1,2}$, James D. Brenton ${ }^{1,2}$, Carlos Caldas ${ }^{1,2,4}$, Simon Pacey ${ }^{2,4}$, Richard Baird $^{2,4 *}$, Nitzan Rosenfeld ${ }^{1,2 *}$

${ }^{1}$ Cancer Research UK Cambridge Institute, Li Ka Shing Centre, Robinson Way, Cambridge ${ }^{2}$ Cancer Research UK Cambridge Centre, Cambridge CB2 ORE

${ }^{3}$ Clinical Trials Unit, Clínic Institute of Haematological \& Oncological Diseases, Hospital Clínic de Barcelona, IDIBAPs, Carrer de Villarroel, 170 Barcelona 08036

${ }^{4}$ Department of Oncology, University of Cambridge

${ }^{*} \mathrm{RB}$ and NR contributed equally.

Please send correspondence to NR at nitzan.rosenfeld@cruk.cam.ac.uk.

\section{Key points}

- cfDNA is released predominantly by cell death into the bloodstream, though active secretion may play a role. Since the discovery of fetal cfDNA in the maternal circulation, cfDNA analysis has been rapidly implemented in clinical practice for non-invasive prenatal testing.

- Mutations were first detected in cfDNA over two decades ago, and interest in ctDNA as a non-invasive cancer diagnostic has increased dramatically with the development of molecular methods that permit the sensitive detection and monitoring of multiple classes of mutation.

- ctDNA may have utility at almost every stage of cancer patient management, including: diagnosis, minimally invasive molecular profiling, treatment monitoring, detection of residual disease, and identification of resistance mutations. ctDNA analysis may be broadly considered as a tool for both quantitative analysis of disease burden and for genomic analysis.

- The identification of ctDNA in individuals prior to a cancer diagnosis, and in pre-symptomatic individuals, suggests the possibility of ctDNA analysis as a 
tool for earlier diagnosis or screening. Non-invasive cancer classification or sub-typing has also emerged as a possibility, though for early detection, both technical and biological factors introduce challenges to the detection of mutant DNA in plasma and its interpretation.

- Monitoring multiple mutations in parallel can enhance sensitivity for ctDNA detection, can be used to assess clonal evolution of patients' disease, and may identify resistance mutations before clinical progression is observed.

- ctDNA analysis is beginning to transition from the research setting into the clinic. The US Food and Drug Administration and the European Medicines Agency have approved ctDNA tests for specific indications in the absence of evaluable tumour tissue. Analysis of gene panels in plasma has now become available as a potential clinical tool. Larger studies are underway to establish the overall performance and clinical utility of such assays when a tumour biopsy is not available for analysis.

- Potential applications of ctDNA have been demonstrated by a number of proof-of-principle studies. Prospective clinical trials are beginning to assess the clinical utility of ctDNA analysis for molecular profiling and disease monitoring. Increasing acceptance of ctDNA is enabling the field to move from exploratory ctDNA studies towards clinical trials where ctDNA is guiding decision making.

- In order to fully exploit the potential utility of liquid biopsies, it is essential that the biology of cfDNA and ctDNA is explored further. Mechanisms of release and degradation, and the factors that affect the representation of ctDNA in plasma, are poorly understood. The nature of ctDNA will be clarified through both large, well-annotated clinical studies, and through in vivo studies, where variables may be controlled. 


\section{Preface}

Improvements in genomic and molecular methods are expanding the range of potential applications for circulating tumour DNA (ctDNA), both in a research setting and as a 'liquid biopsy' for cancer management. Proof-of-principle studies have demonstrated the translational potential of ctDNA for prognostication, molecular profiling, and monitoring. The field is now at an exciting transitional period where ctDNA analysis is beginning to be applied clinically, although there is still much to learn about the biology of cell-free DNA. This is an opportune time to appraise potential approaches for ctDNA analysis, and to consider their applications in personalised oncology and in cancer research.

The presence of fragments of cell-free nucleic acids in human blood was first described in 1948 by Mandel and Métais ${ }^{1}$. The origins and characteristics of cell-free DNA (cfDNA) were studied intermittently in subsequent decades ${ }^{2}$. In healthy individuals, cfDNA concentration tends to range between $1-10 \mathrm{ng} / \mathrm{millilitre}(\mathrm{ml})$ in plasma ${ }^{3,4}$. Raised cfDNA levels were first reported in the serum of cancer patients in 19775; cfDNA concentration can also be raised by other physiological conditions or clinical scenarios, such as acute trauma ${ }^{6}$, cerebral infarction ${ }^{7}$, exercise ${ }^{8}$, transplantation $^{9}$, and infection ${ }^{10}$. Furthermore, the identification of fetal DNA sequences in maternal plasma by Dennis Lo and colleagues in $1997^{11}$ has led to multiple applications of cfDNA in prenatal medicine including sex determination ${ }^{12}$, identification of monogenic disorders ${ }^{13}$, and non-invasive prenatal testing (NIPT) for aneuploidies such as Down's Syndrome (trisomy 21). NIPT was first demonstrated in 2007 by Lo et al. ${ }^{14}$ and has moved rapidly into widespread clinical use ${ }^{15,16}$.

In 1989, Stroun, Anker et al. identified that at least some cfDNA in the plasma of cancer patients originates from cancer cells ${ }^{2,17}$. In 1991, Vogelstein, Sidransky and colleagues showed that DNA from urinary sediments (cell pellets) from patients with invasive bladder cancer carried mutations in TP53, setting the stage for the use of genomic analysis methods in liquid biopsy applications ${ }^{18}$. KRAS mutations were soon found in stool or sputum that matched mutations from colorectal ${ }^{19}$, pancreatic ${ }^{20}$ or lung $^{21,22}$ cancers. In 1994, mutated KRAS sequences were first reported to be 
detected in plasma cfDNA of patients with pancreatic cancer using polymerase chain reaction $(\mathrm{PCR})$ with allele-specific primers ${ }^{23}$. For each patient, the KRAS mutation found in the plasma was identical to that found in the patient's tumour, thereby confirming that the mutant DNA fragments in plasma were of tumour origin. Mutations in cfDNA are highly specific markers for cancer, which gave rise to the term circulating tumour DNA (ctDNA).

In the following decades, ctDNA was explored as a prognostic or predictive marker $^{24,25}$ and for cancer detection ${ }^{26}$. Such studies confirmed the potential of ctDNA, though the levels of ctDNA in different clinical contexts were not yet accurately defined. These studies nonetheless could demonstrate potential clinical applications, for example detection of KRAS mutations in plasma as a potential prognostic factor in colorectal cancer ${ }^{27}$. The introduction of a digital PCR (dPCR) method in 1999 by Vogelstein and Kinzler enabled the accurate identification and absolute quantification of rare mutant fragments ${ }^{28}$. A modification of this technique using beads in emulsions ${ }^{29}$ and flow cytometry allowed the quantification of the mutant allele fraction of cancer mutations in the plasma of patients with different stages of colorectal cancer $^{30}$. Diehl, Diaz et al. then showed in 2008 that ctDNA is a highly specific marker of tumour dynamics, and may be able to indicate residual disease ${ }^{31}$. In parallel, allele-specific PCR and other methods were devised and tested for their ability to identify epidermal growth factor receptor (EGFR) mutations in serum or plasma of lung cancer patients ${ }^{32}$, following the elucidation of the role of such mutations in predicting response to treatment with molecularly targeted inhibitors $^{25,33,34}$.

The development of next generation sequencing-based technologies has facilitated the interrogation of the genome at a broader scale. In 2012, deep sequencing of multiple genes in cfDNA was demonstrated using panels of tagged amplicons, which allowed the identification of mutations directly in the plasma of cancer patients, and monitoring of multiple tumour-specific mutations in a single assay ${ }^{35}$. This method was subsequently applied to monitor ctDNA in a cohort of patients with metastatic breast cancer $^{36}$. Shortly thereafter, whole-genome sequencing (WGS) of plasma cfDNA was 
first shown to identify tumour-derived chromosomal aberrations ${ }^{37}$, focal amplifications $^{38}$ and gene rearrangements ${ }^{39}$, and hybrid-capture sequencing was introduced as a non-invasive method to analyse the evolving genomic profile of mutations in cancer across the entire exome ${ }^{40}$.

There is a clear clinical need for novel diagnostic and molecular tools in oncology (Box 1). Conventional sampling methods such as needle biopsies are subject to procedural complications in up to one in six biopsies ${ }^{41}$, difficulty in obtaining sufficient material of adequate quality for genomic profiling (reported failure rates range from $<10 \%$ to $>30 \%$ of cases $)^{42,43}$, and sampling biases arising from genetic heterogeneity ${ }^{44-48}$. Detection and monitoring of disease often relies on body fluidbased markers that often lack specificity ${ }^{49}$, and imaging which exposes patients to ionising radiation ${ }^{50}$ and has limited resolution (in both time and space). Recent advances in ctDNA research highlight the potential applications of liquid biopsies at each stage of patient management (Fig. 1a). These potential applications primarily arise from two types of information obtainable through ctDNA analysis: quantification of disease burden, and genomic analysis of cancer (Fig. 1b). These may be combined and/or leveraged through serial sampling in order to monitor disease burden and clonal evolution.

The increasing availability and reliability of techniques for PCR and high-throughput sequencing are facilitating novel high-sensitivity applications, the generation of large clinical datasets, and a better understanding of the origin of both cfDNA and ctDNA. This Review will highlight and explore recent advances in the field and the implications for oncology.

\section{cfDNA and ctDNA biology}

\section{Characteristics of cfDNA and ctDNA}

cfDNA is thought to be released from cells mostly through apoptosis and necrosis, and possibly also active secretion ${ }^{2,51-54}$. Outside of the blood circulation, cfDNA has been detected a variety of body fluids including urine ${ }^{55-58,59}$, cerebrospinal fluid $(\mathrm{CSF})^{60-63}$, pleural fluid ${ }^{64}$ and saliva ${ }^{65}$. Genetic and epigenetic modifications of cfDNA 
molecules reflect the genome or epigenome of the cell of origin ${ }^{66-68}$ (Fig. 2). Methylation analysis has revealed that the majority of cfDNA in plasma is released from haematopoietic cells in healthy individuals ${ }^{67-69}$. These have been suggested to be the source of cfDNA release following intense exercise ${ }^{70}$. Observational studies have determined the half-life of cfDNA in the circulation as between 16 minutes and 2.5 hours $^{31,71-73}$, which allows ctDNA analysis to be considered as a 'real-time' snapshot of disease burden. Other observational studies indicate that cfDNA is cleared from the circulation via nuclease action ${ }^{72,74}$ and renal excretion into the urine ${ }^{55,58,75}$. cfDNA uptake in the liver and spleen, followed by degradation by macrophages, may also contribute ${ }^{30,76}$. The stability of individual fragments in the circulation may be increased through association with cell membranes, extracellular vesicles or proteins ${ }^{2}$.

Nearly two decades ago, the modal size of cfDNA was determined using gel electrophoresis as $\sim 180$ base pairs (bp), indicating that cfDNA was likely to be nucleosome-associated ${ }^{77}$. Sequencing-based approaches have since refined this measurement, by identifying a prominent peak at $166 \mathrm{bp}^{78,79}$, corresponding to the length of DNA wrapped around a nucleosome ( 147bp), plus linker DNA associated with histone $\mathrm{H} 1$. Fragment size traces of cfDNA show a $10 \mathrm{bp}$ ladder pattern ${ }^{54,75}$, ostensibly caused by nucleases cleaving the DNA strand at periodically exposed sites with each turn of the DNA double-helix. The fragmentation patterns of cfDNA differ between plasma and urine ${ }^{75}$, potentially contributed to by a higher nuclease activity in urine ${ }^{80}$.

ctDNA molecules are shorter than non-mutant cfDNA in plasma, demonstrated by $\mathrm{PCR}^{4,81}$ and sequencing ${ }^{68,82}$. Animal xenograft experiments ${ }^{79,81-83}$ provide an elegant means to interrogate ctDNA, since any human DNA sequences must have originated from the tumour xenograft. The modal length of ctDNA fragments has been measured in a rat xenograft model as between $134-144 \mathrm{bp}^{82}$, though the cause of this shortening is not clear. Shortening of fragments is also observed in fetal cfDNA relative to maternal $\operatorname{cfDNA}^{78}$, and between non-haematopoetically-derived vs. haematopoietically-derived cfDNA fragments in transplant patients ${ }^{84,85}$. Differences in 
nucleosome wrapping or nuclease action between haematopoietic cells, which contribute most to the cfDNA pool, and other tissues may play a role. Long cfDNA fragments (>1000bp) have been observed in healthy individuals using long-read sequencing techniques ${ }^{86}$, and may be released into the circulation in association with exosomes $^{51,52}$, or by tumour cells via necrosis ${ }^{53}$. Current extraction methods often poorly recover these long fragments ${ }^{87,88}$ (Box 2). Commonly used library preparation methods introduce further biases: single-stranded DNA (ssDNA) library preparation ${ }^{89}$ can recover DNA fragments with damaged ends, and when applied to cfDNA ${ }^{66,90}$ uncovered a large proportion of fragments shorter than 100 bases. Diverse extraction and sequencing methods may therefore yield complementary data. Combining those with histological analysis of corresponding tissue samples could provide new insights into the biological determinants of cfDNA fragmentation, and the biological origins of cfDNA.

\section{Physiological and pathological considerations}

cfDNA has been proposed as a ligand for Toll-like receptor 9 (TLR9) ${ }^{91,92}$, a sensor of exogenous DNA fragments, found primarily in tissues rich in immune cells. In mice, obesity-related adipocyte degeneration was shown to release cfDNA, which contributed to macrophage accumulation via TLR9 activation, leading to adipose tissue inflammation and insulin resistance ${ }^{92}$. Another study has suggested that cfDNA may inhibit pro-apoptotic caspases via TLR9-depedent signalling ${ }^{91}$, which could imply a potential immunomodulatory role for cfDNA.

In vitro experiments suggest that cfDNA may be internalised by cells ${ }^{2,93,94}$, raising the possibility that cfDNA molecules could mediate the horizontal transfer of genes or DNA. One report showed in vitro transformation of NIH-3T3 mouse cells that were in contact with samples of plasma from patients with KRAS-mutant colorectal cancers, despite being separated to avoid tumour cell contamination ${ }^{95}$. Another study demonstrated integration of ctDNA into the nuclear DNA of recipient cells, and suggested that this may occur through non-homologous end-joining ${ }^{93}$. A similar phenomenon has been observed with mitochondrial DNA ${ }^{96}$. Overall, it is clear that 
there is a lot to learn about the biology of cfDNA and ctDNA, which could have an important impact on their potential applications in oncology.

\section{Approaches for ctDNA analysis}

Analysis of ctDNA ranges in scale from single mutations to whole-genome analyses (Fig. 3; See Table 1 for a comparison of selected techniques). Appropriately designed assays for individual mutations can achieve high sensitivity using a simple workflow. Allele-specific PCR methods ${ }^{97,98}$ have been applied since the mid-2000s for detection of hot-spot mutations in serum and plasma ${ }^{25,33,34,99}$, and some assays are available as kits that are approved for clinical use ${ }^{158,160}$, but have limited analytical sensitivity. dPCR assays on microfluidic platforms are quantitative and highly sensitive, and are used extensively for quantifying ctDNA levels ${ }^{29,30,32,57,100-102}$. Improved detection at selected loci has been demonstrated by methods such as single-base extension ${ }^{103}$ or enrichment for mutant alleles by electrophoretic methods ${ }^{131,132}$, nuclease activity ${ }^{104}$ or modified $\mathrm{PCR}^{105-107}$. The multiplexing capacity of such assays, that rely on differential binding affinities of mutant and wild type alleles, and for the most part require primers or probes that are specific to a defined mutation or targeted locus, is limited. These are, therefore, generally suited to investigating a small number of mutations, and are often applied to analysis of cancer hot-spot mutations. If samples need to be split into multiple reactions, this increases sampling error, and may impair the overall performance of an assay for very low copy numbers of mutant DNA.

In order to interrogate a larger number of loci, targeted sequencing using PCR amplicons or hybrid-capture have been employed ${ }^{35,40,108,109}$. Regions for sequencing may range from individual exons of interest (kilobases), to the entire exome ( 50 megabases). Current off-the-shelf panels for gene sequencing can detect mutations with an allele fraction greater than $1 \%{ }^{46,110}$. By reducing background error rates of sequencing, for example by molecular barcoding (Fig. 3) or multiple replicates (Fig. 4), ctDNA can be detected at allele fractions below $0.1 \%{ }^{111-114}$ (Table 1). Ampliconbased assays that have been optimised for the purpose of ctDNA analysis can target 
dozens to hundreds of amplicons across multiple kilobases ${ }^{35,112,114}$. Hybrid-capturebased approaches can increase the genomic region studied to dozens or hundreds of kilobases ${ }^{108,109,111,115}$. The sensitivity for ctDNA detection can be further enhanced, even with limited amounts of input material, by using multiplexed patient-specific panels in combination with targeted sequencing methods ${ }^{35,111}$ (Fig. 4).

Amplifications and deletions may be identified through low-depth $(\sim 0.1 \mathrm{x}$ coverage) sequencing of the whole genome, with comparison of the relative number of sequencing reads between equally sized genomic regions across a sample or between samples and controls ${ }^{39}$. Such shallow WGS (sWGS) has been employed to detect fetal aneuploidies ${ }^{38}$, and it can also be used to detect cancer-specific copy number alterations ${ }^{37,39,116}$. sWGS has a limit of detection of between $5 \%-10 \%$ mutant allele fraction (Table 1), and so has limited sensitivity for profiling earlier stage disease. If molecular profiling of a small number of recurrent copy number alterations is desired, higher sensitivity may be achieved through targeted sequencing of single nucleotide polymorphisms, which may detect copy number alterations as low as $0.5 \%{ }^{117}$.

The limit of detection for assays will vary based on whether the individual's disease status, and tumour mutations, are already characterised. Tumour burden in plasma has often been assessed by quantifying mutations (or other alterations) that were previously identified in the patient's tumour sample $e^{30,31}$. For mutation calling across a panel of genes or hotspots, the risk of false positives increases with the size of the panel due to multiple hypothesis testing, and filters need to be applied to increase specificity, which erodes sensitivity for rare variants. Prior knowledge of the mutation profile (e.g. from tumour sequencing data) enables the detection of known patientspecific mutations above the background error rate, as opposed to calling mutations de novo ${ }^{35}$. Thus, sequencing-based assays can be used as sensitive and quantitative tools for ctDNA measurement and monitoring, in addition to their use for mutation profiling $^{35,36,108}$ (Fig. 1b). 
ctDNA can be quantified using different metrics, such as mutant allele concentration (i.e. copies per $\mathrm{ml}$ ) or mutant allele fraction ${ }^{118}$. Each of these metrics would be affected in a different way by analytical, pre-analytical, and physiological characteristics. For example, metabolic changes to the rate of cfDNA turnover would affect the concentration of mutant alleles more than the mutant allele fraction, whereas pre-analytical factors affecting release of germline DNA from blood cells would reduce the mutant allele fraction to a greater extent. Analysis of ctDNA (both fraction and concentration) ${ }^{118}$, as well as total cfDNA and cfDNA fragmentation ${ }^{3,119}$, could therefore provide complementary information, and may have advantages in different applications or in combination.

\section{ctDNA detection across cancer stages}

ctDNA relates to stage and prognosis

The concentration of ctDNA in plasma has been shown to correlate with tumour size $^{79,120}$ and stage ${ }^{121}$. A study of 640 patients with various cancer types and stages $^{121}$ found a 100 -fold increase in median ctDNA concentration between patients with Stage I and Stage IV disease. Measuring individual tumour mutations in each patient, patients with Stage I disease had fewer than 10 copies per $5 \mathrm{ml}$ of plasma. In sharp contrast, patients with advanced prostate, ovarian and colorectal cancers had a median concentration of $100-1,000$ copies per $5 \mathrm{ml}$ of plasma. ctDNA levels vary greatly even within patients with the same type and stage of disease. This variability in ctDNA concentration is partially explained by differences in extent of metastatic spread or disease burden. In a recent report that compared ctDNA levels with tumour volume assessed by imaging in patients with relapsed high-grade serous ovarian cancer, ctDNA levels and disease volume were significantly correlated ${ }^{118}$. Mutant alleles in plasma increased in fraction by approximately $0.08 \%$, and in concentration by 6 mutant copies per millilitre of plasma, for every $\mathrm{cm}^{3}$ of disease ${ }^{118}$. Notwithstanding these correlations, substantial variation in ctDNA concentration may arise from inter-individual differences. For example, poor tumour vascularisation could hamper ctDNA release into the bloodstream, or conversely, could promote ctDNA release via producing hypoxia and cell death. Histological differences could forseeably influence both the rate and type of cell death. Patients with primary brain 
tumours have very low levels of ctDNA, with a median concentration for individual mutations of less than 10 copies per $5 \mathrm{ml}$ of plasma ${ }^{121}$, while the fraction of tumour DNA in CSF was found to be significantly higher ${ }^{61,62,121}$. Although not directly proven, the blood-brain barrier has been suggested to impede the movement of cfDNA fragments into the circulation ${ }^{61,62,121}$.

The relationship between ctDNA levels and cancer stage suggests prognostic utility for ctDNA. Patients with detectable ctDNA have been shown to have worse survival outcomes than those without ${ }^{27,122-126}$. In one of the earliest examples in the field, the 2-year overall survival rate for patients with colorectal cancer who had detectable ctDNA was $48 \%$, as opposed to $100 \%$ for patients without ${ }^{27}$. In patients with detectable ctDNA, it has been found to be a more significant prognostic predictor than commonly used tumour markers ${ }^{36,118}$, where an increasing concentration of ctDNA correlates with poorer clinical and radiological outcomes ${ }^{36,118,121,127,128}$. For example, in patients with metastatic breast cancer, a significant inverse correlation was shown between ctDNA concentration and overall survival up to $2000 \mathrm{copies} / \mathrm{ml}$, with a uniformly poor prognosis above this level ${ }^{36}$. In addition to ctDNA levels, mutational patterns identified in ctDNA (Fig. 1b) can help group patients into molecular subtypes with different prognosis ${ }^{129}$.

\section{Earlier diagnosis of disease}

Diagnosing cancer at an earlier stage, particularly before metastatic spread, may allow earlier intervention and could improve survival ${ }^{130}$. A number of studies have demonstrated the potential for non-invasive early diagnosis. Mutations have been detected in saliva and plasma from individuals up to two years prior to cancer diagnosis $^{21,131}$, and there have been reports of incidental pre-symptomatic detection of cancers in pregnant women who underwent NIPT ${ }^{68,132,133}$, as WGS can identify copy number alterations of both fetal and tumour origin. Screening in asymptomatic populations introduces risks of over-diagnosis and false positives; implementation could therefore be explored in stages, and a first step could involve the use of ctDNA for earlier diagnosis of disease in symptomatic individuals, who at present may undergo lengthy investigation procedures. 
In a survey across several cancer types, ctDNA was detected in $82 \%$ of patients with Stage IV disease, which fell to $47 \%$ for patients with Stage I disease ${ }^{121}$. The method they applied was benchmarked as being able to detect one copy of an individual cancer mutation per $5 \mathrm{ml}$ of plasma ${ }^{121}$. Using a sequencing gene panel that targeted a median of four mutations per patient, ctDNA was detected in $50 \%$ of patients with stage I non-small cell lung cancer (NSCLC) ${ }^{108}$. Targeting known tumour mutations in plasma using ddPCR assays in early-stage breast cancer showed a sensitivity of $93.3 \%{ }^{134}$. A sWGS method adapted from an NIPT assay was recently shown to detect $6 / 16(37.5 \%)$ cases of early ovarian cancer ${ }^{135}$, though this approach may not perform as well in other cancer types with fewer copy number alterations. Together, these studies outline the possibility, and the challenge, of detection of ctDNA in early stage disease.

If analysis is performed on a few millilitres of plasma containing only a few thousand copies of the genome, increasing the analytical sensitivity beyond the range of $\sim 1 / 1000$ may not produce any sensitivity benefit since at low allele fractions, it becomes increasingly likely that the mutation of interest may not be found due to sampling noise. One approach may be to collect greater volumes of plasma (and more cfDNA) through methods such as plasmapheresis or implanted devices containing materials that bind cfDNA; similar approaches have been tested for enhancing the yield of circulating tumour cells (CTCs) ${ }^{136}$. Given these challenges, it is important that pre-analytical factors surrounding the collection, processing, and extraction of cfDNA are optimised (Box 2). Alternatively, for some cancer types, other minimally invasive samples may have a higher tumour DNA content, such as urine for bladder cancer ${ }^{57}$ or stool for colorectal cancers ${ }^{19}$; or cytological specimens such as cervical smears ${ }^{137}$, uterine lavage ${ }^{138}$, or oesophageal brushings ${ }^{139}$ for gynaecological or oesophageal cancers, respectively (Fig. 3c). For cancers with a viral aetiology, e.g. nasopharyngeal carcinoma or cervical cancer, detection of the cancer-associated viral DNA that may be present in body fluids in many more copies than tumour DNA can enhance the identification of individuals with early stage disease or pre-malignant lesions with a high risk for cancer ${ }^{140,141}$. 
Technical and biological advances facilitate enhanced mutation detection: the finding that $\operatorname{ctDNA}$ is shorter than $\operatorname{cfDNA}^{4,81,82}$ suggests the utility of experimental or in silico size selection (Fig. 3d). Where the input amount of plasma or DNA is limiting, assays that interrogate multiple mutations in the same reaction (through broad genomic coverage or by patient-specific multiplexed panels) may produce a higher overall sensitivity for detecting the presence of any $\operatorname{ctDNA}{ }^{35,36,108,110,111}$, compared to focused analysis of individual loci (Fig. 4). Taken to a (currently impractical) extreme, ultradeep sequencing of the entire genome could in the future allow sensitive detection of cancer even from small volumes of plasma ${ }^{37}$ (or other fluids).

Although technical advances may improve sensitivity for ctDNA analysis further, biological and genomic factors may eventually become limiting. For confident de novo cancer detection using ctDNA, detected alterations should have a high positive predictive value for cancer. However, mutations known to be associated with cancer (e.g. in TP53, KRAS, and Notch pathway genes) have been found at low levels in skin biopsies of healthy individuals ${ }^{142}$. If non-tumourigenic clones were to increase to sufficient size and release mutated cfDNA, they could introduce biological noise. Clonal haematopoiesis with leukaemia-associated mutations has been observed in $10 \%$ of individuals older than 65 years of age, though the absolute risk of conversion to haematologic cancer is $1 \%{ }^{143}$. Genomic alterations known to be associated with cancer have been found in plasma from healthy individuals ${ }^{111,115,144,145}$. Clinical outcomes for apparently healthy individuals in whom mutant DNA is detected in plasma should be characterised in order to understand the biological and clinical implications of such findings.

\section{Cancer localisation}

Tissue-of-origin information from liquid biopsies might be able to aid cancer localisation, for example, for cancers of unknown primary ${ }^{146}$. Methylation and nucleosome occupancy patterns in cfDNA have been found to encode tissue- and cell-specific information ${ }^{66-68,147}$ : in one case, a pregnant woman had chromosomal abnormalities detectable by NIPT, and so tissue-specific methylation signals in 
plasma were studied, enabling quantification of the relative contributions of each tissue to the total cfDNA pool ${ }^{68}$. This revealed an increased contribution of cfDNA from B-lymphocytes, consistent with a diagnosis of follicular lymphoma ${ }^{68}$. While it may be possible in the future to deduce the tissue type of origin of a cancer in this fashion, it remains to be seen whether the site or sites of metastatic spread can be determined through a measurable increase in tissue-specific cfDNA signal.

\section{Non-invasive molecular profiling}

Analysis of heterogeneity

The extent of genetic heterogeneity has been confirmed over recent years as multiregional sequencing studies have demonstrated clear differences in mutation profiles between different tumour regions in the same patient ${ }^{148,149}$ and between different specimens from primary and metastatic sites ${ }^{150}$. Although the potentially confounding effects of heterogeneity are recognised, it is often neither feasible nor desirable to perform multiple tumour biopsies on patients to try to account for this. Analysis of an individual biopsy might not accurately reflect the genomic architecture of a patient's cancer, introducing bias to the selection and efficacy of personalised medicines. Furthermore, in a recent study of patients with lung cancer treated with an EGFR inhibitor, the tumour $E G F R^{T 790 M}$ allele fraction correlated with the degree of tumour shrinkage ${ }^{151}$, suggesting that the current paradigm of treatment selection based on mutation presence or absence alone may be suboptimal.

Liquid biopsies sample ctDNA released from multiple tumour regions, and may thereby reflect both intratumour heterogeneity ${ }^{45,47,62}$ and spatially separated disease foci $^{48,152-154}$. While individual tumour biopsies from different tumour regions may differ in mutation profile due to intratumour heterogeneity ${ }^{155,156}$, ctDNA analysis has detected mutations missed in corresponding tissue samples ${ }^{45,48,157,158}$. Multi-region tumour sequencing data show that stem mutations (shared by all tumour regions) show a higher allele fraction in plasma compared to private mutations ${ }^{47,48}$. Therefore, for tracking tumour burden in plasma, stem mutations would provide the most reliable detection. Alternatively, tracking a large set of mutations may compensate for potential biases of individual private mutations. 


\section{Hotspot mutations and gene panels}

By comparing mutation detection in plasma against matched tumour samples, the sensitivity of ctDNA analysis has been estimated in retrospective studies as between $65 \%-98 \%{ }^{100,102,119,154,157,159-161}$. For profiling specific loci, for example in order to stratify patients for matched molecular therapies, international studies have begun to demonstrate that large-scale testing is feasible and may be standardised, although the use of assays with limited analytical sensitivity resulted in low detection rates of ctDNA $^{162-164}$. Using assays developed specifically to detect low levels of ctDNA ${ }^{3}$, a blinded prospective study demonstrated sensitivity for KRAS and BRAF mutations in metastatic colorectal cancer of $92 \%$ and $100 \%$, respectively, with concordance rates of $96 \%$ and $100 \%$ for each ${ }^{119}$.

Considerable attention has been devoted to analysis of EGFR mutations in patients with $\mathrm{NSCLC}^{34,99}$, as it is often challenging to obtain tissue biopsies to help inform treatment ${ }^{41,42}$. Meta-analysis of 27 selected studies published between 2007-2015, comprising altogether nearly 4000 patients, resulted in a pooled sensitivity of $60 \%$ and specificity of $94 \%$ for detection of EGFR mutations in plasma or serum, with a variety of methods ${ }^{34}$. In a phase IV study of the EGFR inhibitor gefitinib, mutation status was compared between tumour and plasma samples from 652 patients. The sensitivity and specificity for detecting mutations in plasma were determined as $65.7 \%$ and $99.8 \%$, respectively ${ }^{164,165}$, likely affected by the limited analytical sensitivity of the PCR-based method used ${ }^{166}$, as the version of the kit used in that study had a limit of detection (at $\geq 95 \%$ analytical sensitivity) of $1.64 \%$ and $1.26 \%$ for EGFR deletions and L858R mutations, respectively ${ }^{164}$. Low rates of concordance of $E G F R^{T 790 M}$ status were also observed in a recent phase III trial of osimertinib ${ }^{167}$, in which tissue testing was compared to plasma using an allele-specific PCR assay for ctDNA analysis that has a limit of detection (with $\geq 95 \%$ analytical sensitivity) of 100 copies of $E G F R^{T 790 M}$ per $\mathrm{ml}$ of plasma ${ }^{168}$. Using methods with greater analytical sensitivity, higher concordance rates were obtained ${ }^{157,169}$. The EGFR ${ }^{T 790 M}$ mutation confers resistance to gefitinib and erlotinib, and frequently emerges following initial treatment with those EGFR tyrosine kinase inhibitors ${ }^{33,170,171}$. In plasma, the 
sensitivity for detecting this mutation was lower than for $E G F R^{L 858 R}$ and $E G F R^{\mathrm{Ex} 19 \mathrm{del}}$ ( $70 \%$ vs. $\geq 90 \%$ ), which occur earlier in the development of disease ${ }^{157,169}$. Initial data suggests that this may be due to heterogeneous presence of resistance mutations at disease relapse ${ }^{157}$.

In retrospective analyses, despite limited concordance rates of EGFR mutation status between plasma and tumour samples observed in some studies, response rates for patients who were plasma positive for mutations in EGFR were similar to response rates of patients who were tissue positive $e^{157,164,165,167,169}$. Data showing the response of patients treated solely on the basis of ctDNA analysis are starting to emerge: patients who were treated with osimertinib based on detection of $E G F R^{\mathrm{T} 790 \mathrm{M}}$ in plasma, and had response rates similar to the response rates of patients treated based on tissue analysis ${ }^{172}$. Interestingly, objective responses were also seen in patients with very low allele fractions of mutant $E G F R^{\mathrm{T790M}}$ in plasma $(<0.5 \%)^{172}$.

At present, the European Medicines Agency (EMA) and the US Food and Drug Administration (FDA) approve the use of information from ctDNA analysis to help select patients with EGFR-mutant NSCLC for gefitinib (EMA) ${ }^{173}$, erlotinib (FDA) ${ }^{168}$ or osimertinib (EMA and FDA) $)^{168,174}$ therapy in the event that a tumour sample is not evaluable. This could offer a pragmatic solution to provide molecular profiling information for patients, while avoiding repeat biopsies for some individuals. Current recommendations ${ }^{168,174}$ state that if liquid biopsies are carried out in advance of a tumour biopsy, ctDNA detection may abrogate the need for tissue biopsy, but if ctDNA analysis is negative, a tissue biopsy may still provide valuable genomic information.

Molecular profiling using ctDNA may have particular utility for stratifying patients in 'basket trials', which enrol patients independent of tumour histology, or 'umbrella trials', which assign patients to multiple investigational drugs or treatment options ${ }^{175}$. For example, a 54-gene panel detected ctDNA in 58\% of patients across multiple cancer types ${ }^{176}$. Of the patients with alterations, $71.4 \%$ had at least one mutation actionable by an FDA-approved drug ${ }^{176}$. This panel is being used to test the 
feasibility of matching patients with different metastatic cancer types to targeted therapies in a prospective clinical trial ${ }^{177}$. In another study presented at the 2016 Molecular Analysis for Personalised Therapy meeting, a 34-gene panel identified mutations in $79 \%$ of 174 patients with NSCLC, allowing 28 patients $(17 \%)$ to receive personalised treatment based on ctDNA molecular profiling ${ }^{178}$. Personalised therapy selection presents challenges: even if mutations are successfully detected using ctDNA in patients, an efficacious molecularly targeted agent may not exist. However, data from a prospective clinical trial presented at the 2016 Molecular Analysis for Personalised Therapy meeting demonstrated that selecting therapies based on genomic analysis can improve outcomes for cancer patients, even when patients with well-established actionable targets (for which approved drugs are available) were excluded $^{179}$.

Improvements in the analytical sensitivity of molecular profiling tools could further increase detection and concordance rates or allow for sensitive multiplexed analysis (Table 1), though biological factors and heterogeneity may reduce sensitivity in some cancer types and stages ${ }^{121,157,180}$. The utility of ctDNA should, therefore, be assessed for different clinical indications. However, benchmarking ctDNA against individual tumour biopsies may be confounded by sampling error, as rare private mutations may be sampled in the biopsy, but release insufficient ctDNA into the bloodstream to be detectable.

\section{Structural variants}

Copy number alterations can be detected in cfDNA using WGS ${ }^{38,181-183}$, ampliconbased $^{116,184,114}$, and hybrid-capture approaches ${ }^{40,108,160,185}$. In patients with hepatocellular carcinoma, WGS was able to identify amplifications and deletions in plasma matching those identified in tumour tissue ${ }^{38,186}$. Heterogeneous copy number changes were also identified in a patient with synchronous breast and ovarian cancers, as copy number changes unique to each cancer were detected in plasma ${ }^{38}$. In a study of 80 patients with prostate cancer, androgen receptor $(A R)$ copy number gain prior to abiraterone therapy predicted a worse overall survival, thus identifying patients with primary resistance ${ }^{187}$. For patients with advanced disease, sWGS may 
provide a relatively cost-effective measure of ctDNA level that is applicable across cancer types. This approach may have utility as a sample screening step in a ctDNA analysis workflow ${ }^{116}$, where high-burden patient samples are triaged for exome sequencing ${ }^{40}$.

Chromosomal rearrangements in plasma can be identified through both $\mathrm{WGS}^{37}$ and hybrid-capture sequencing approaches ${ }^{39,188}$, though the latter may be more economical due to the depth of coverage needed to confidently identify a rearrangement. In one study of patients with prostate cancer, sWGS was able to detect a deletion on chromosome 21 in 5 patients, though higher-depth capture sequencing was necessary to identify a rearrangement between exon 1 of TMPRSS2 and exon 3 of $E R G^{39}$.

\section{Longitudinal monitoring}

Monitoring response

The short half-life of cfDNA, ${ }^{31,71-73}$ as well as the ease and reduced risk of repeating liquid biopsies relative to imaging ${ }^{50}$ or tissue biopsies ${ }^{41}$, enables liquid biopsies to be used for real-time monitoring of cancer burden in response to therapy. Studies monitoring patients during treatment have shown that ctDNA dynamics correlate with treatment response $\mathrm{e}^{31,35,36,108,126}$, and may identify response earlier than clinical detection ${ }^{36,189,190}$. In patients with breast cancer, ctDNA showed the greatest range in concentration and provided the earliest measure of response to chemotherapies, as well as the earliest indication of impending relapse compared to imaging and other blood-based cancer markers, such as CTCs and Cancer Antigen 15-3 (CA 15-3, also known as MUC1) ${ }^{36}$. In relapsed ovarian cancer, pre-treatment ctDNA levels and the extent of ctDNA decrease after chemotherapy initiation were significantly associated with time to progression, and were more informative than levels of CA 125 (also known as MUC16) ${ }^{118}$.

A recent study suggested that an early spike in ctDNA levels (allele fractions of BRAF mutations) in the first week following the initiation of immunotherapy in melanoma patients may predict response ${ }^{191}$. This may reflect a transient increase in 
cell death. If these data are confirmed, sampling at early time points could be applied in the clinic as well as in drug development. However, the presence or timing of such spikes in cell death would likely vary based on the pharmacological properties and biological responses to treatments used. An early spike was not observed a few days after initiation of treatment with chemotherapy for patients with colorectal cancer ${ }^{159}$ or with EGFR inhibitor for patients with NSCLC ${ }^{190}$. If analysis of plasma immediately after the start of therapy could reliably detect the destruction of sensitive cancer cells, this raises an exciting possibility that the existence of resistant sub-clones could be identified very rapidly through differential early dynamics of mutations. In the context of immunotherapy, liquid biopsies may provide the opportunity to monitor both ctDNA and the response of the immune system, for example through the analysis of cfDNA released from distinct $\mathrm{T}$-cell clones $^{192}$.

\section{Minimal residual disease and recurrence monitoring}

Following surgery or treatment with curative intent, even in the absence of any other clinical evidence of disease, detection of ctDNA may signal the presence of minimal residual disease (MRD), which could identify patients who may be at a higher risk of relapse. Stratification of patients into high- and low-risk groups would enable adjuvant therapy to be given to patients who stand to benefit most, while sparing lowrisk patients from unnecessary comorbidities and risks of adverse events. In a prospective study of 230 early-stage colorectal cancer patients, assessment of ctDNA at the first follow-up visit after surgical resection indicated that recurrence-free survival at 3 years was $0 \%$ for the ctDNA-positive and $90 \%$ for the ctDNA-negative groups $^{193}$. In a separate study of 55 patients with early-stage breast cancer, assessment of ctDNA showed that detection of ctDNA at first follow-up could also indicate poor prognosis in early-stage breast cancer ${ }^{194}$. Furthermore, stratification based on mutation detection across serial samples improved prediction of relapse, and this and other studies have observed an interval of 7.9-11 months between ctDNA detection and clinical relapse ${ }^{194-196}$, similar to that identified in the metastatic setting $^{36}$. With more sensitive approaches (Fig. 4), earlier identification of clinical relapse should be possible. Combining monitoring for known mutations with molecular profiling assays could allow identification of potential targets for early 
therapeutic intervention - ideally, such intervention would, in future, prevent or postpone overt relapse.

Patient-specific DNA rearrangements identified from sequencing tumour samples can be used to design assays to track tumour burden in plasma ${ }^{196-199}$. Curative surgery could provide an excellent opportunity to obtain tumour DNA that can be sequenced to guide the design of assays for post-operative monitoring. Patient-specific rearrangements may be more readily detected in ctDNA with high sensitivity ${ }^{197,198}$, since rearrangements are less confounded by background noise. One of the challenges of individualised panel design is that sequencing an individual tumour biopsy may not sample every mutation in heterogeneous disease; therefore, sequencing matched body fluid and tumour samples may be desirable for comprehensive mutation profiling. In future, if tumour sequencing becomes routine, monitoring disease using patient-specific panels could become viable, although regulation of such assays will likely be more complex than fixed panels.

\section{Clonal evolution and resistance}

As discussed above, rising or falling ctDNA concentration may provide an indication of treatment effect on overall tumour burden. If multiple tumour mutations are interrogated, then the relative change between each may provide insight into molecular evolution of the patient's cancer ${ }^{40,200,201}$. Ratios between the levels of different mutations in plasma can indicate heterogeneity and may be informative to predict patient response to treatment targeting particular alterations ${ }^{157,160}$. Liquid biopsies have been shown to contain ctDNA from multiple tumour sites ${ }^{45,47,48,62}$, and their analysis has a faster turnaround time than tissue biopsies ${ }^{39,102,188}$, and may be less prone to biases resulting from the analysis of individual tumour biopsies ${ }^{148,149,202}$.

Studies demonstrate that ctDNA can monitor clonal evolution and identify resistance mechanisms to treatment ${ }^{110,158,160,200,203,204}$. Serial ctDNA analysis in patients with colorectal cancer demonstrated the positive selection of mutant KRAS clones during EGFR blockade, which later decline upon the withdrawal of anti-EGFR therapy ${ }^{158,205}$. In patients with NSCLC undergoing treatment with EGFR inhibitors, resistance- 
conferring mutations emerged in plasma ahead of clinical progression ${ }^{170,171}$. Exome sequencing of plasma DNA may identify resistance mechanisms in patients across cancer types ${ }^{40}$, though the sensitivity of exome sequencing currently limits its application to advanced cancer patients where ctDNA levels are high ( $>5 \%$ mutant allele fraction). Design of patient-specific mutation panels ${ }^{35,48,111,206}$ could be a more cost-effective alternative for high-sensitivity monitoring, though may miss subsequent de novo events unless appropriately designed. Serial sWGS analysis also demonstrates highly dynamic copy number adaptations in response to selection pressures, with a mean interval of 26.4 weeks between new amplifications ${ }^{207}$.

Serial liquid biopsies may have particular utility for adaptive or reactive therapy, in which resistance mutations are prospectively identified, and therapy adapted in realtime (Fig. 5). In the clinical research setting, non-invasive monitoring could facilitate a clinical trials that prospectively identify efficacious treatment regimens or drug combinations, and identify resistance mechanisms to novel therapies. In addition, in vitro or in vivo experiments carried out in parallel may provide greater insight into cancer biology. For example, colorectal cancer cell line experiments carried out in parallel with ctDNA analysis showed that resistance mutations may arise from both the selection of pre-existing minor clones, and through ongoing mutagenesis ${ }^{200}$. Another study investigating resistance to a pan-tropomyosin-related kinase (TRK, also known as NTRK) inhibitor in colorectal cancer demonstrated that simultaneous analysis of patient-derived xenografts and liquid biopsies may characterise resistance more comprehensively than plasma alone ${ }^{199}$.

\section{Future directions}

Proof-of-concept studies provide an excellent starting point for larger prospective studies into the clinical utility of ctDNA, and demonstrate that ctDNA may be a useful research tool for drug development, and for the study of intratumour heterogeneity and clonal evolution. Moving forwards, randomised trials comparing ctDNA-guided decision-making against the standard of care would be definitive, and the EMA have outlined good practice guidelines for the design of such trials ${ }^{208}$. Trials to test the clinical utility of ctDNA analysis for treatment monitoring are now being carried 
out $^{209,210}$. In one trial, patients with NSCLC receiving erlotinib are being prospectively monitored, and if resistance mutations emerge in plasma, then additional scans to search for signs of disease progression would be carried out ${ }^{209}$. Another clinical trial aims to demonstrate the efficacy of targeting mutations identified in plasma from patients with advanced breast cancer ${ }^{211}$, which could support the future use of plasma-only mutation profiling and treatment stratification. Together, these studies highlight that the field is moving from exploratory ctDNA studies, towards clinical trials where ctDNA is guiding decision-making.

A better understanding of the origin and biology of cfDNA and ctDNA would aid the implementation of liquid biopsies ${ }^{2}$. The relative contributions of apoptosis, necrosis and active release, particularly at different time points during treatment, should be explored. Our limited understanding of the release and clearance mechanisms of cfDNA hampers interpretation of current studies. Studies of the dynamics and reproducibility of ctDNA measurement in the absence of intervention will become increasingly important as we aim to interpret ctDNA signal in response to treatment. It is also not clear whether all tumour subclones contribute proportionately to the total ctDNA pool, or whether their representation in the bloodstream is biased by other biological factors, such as tumour vascularity or metabolic activity. In vivo cellular

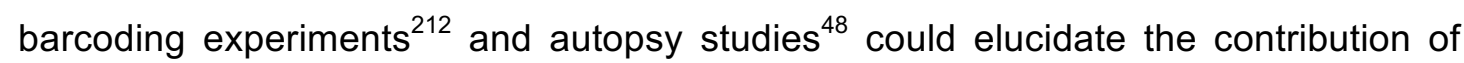
individual subclones, and histological studies may clarify the factors that modulate ctDNA release. The differences in size between cfDNA and ctDNA fragments ${ }^{4,68,81,82}$ suggest that optimising processing and extraction methods (as well as downstream assays) for recovery of selected fragment sizes may provide further improvement to overall performance.

While ctDNA can have greater sensitivity and specificity compared to other circulating biomarkers ${ }^{36}$, taking a multi-marker approach may offer a more comprehensive insight into a patient's disease , $^{3,119,213}$. For example, total cfDNA concentration correlates with disease status ${ }^{3,5}$ and is associated with prognosis ${ }^{214}$. Epigenetic analysis of cfDNA may identify cancer gene hypermethylation ${ }^{27,153}$ or the cell-type giving rise to cfDNA fragments ${ }^{6-68}$, and may provide a window into the 
tumour microenvironment, which usually lacks somatic mutations. Other circulating nucleic acids such as mRNA and microRNA can provide additional layers of information $^{215}$. Targeting multiple types of nucleic acid, with independent mechanisms of release, may increase sensitivity for detection of MRD, for example through the co-isolation of both exosomal RNA and $\operatorname{cfDNA}^{216}$. Actively released nucleic acids may be preferred for the detection of mutations in subclones resistant to therapy, whereas fragments arising from dying cells following the initiation of therapy may identify treatment-responsive subclones. Next, although it may be possible to infer gene expression patterns from $\operatorname{cfDNA}^{217}$, sequencing RNA within exosomes $^{218}$, CTCs $^{219}$ or platelets ${ }^{220}$ could provide more direct evidence. Analysis of cell-free DNA in plasma alongside other fluids such as urine ${ }^{221}$ or $\operatorname{CSF}^{45}$ can provide complementary information. We further echo the suggestion by Gormally, Hainaut and colleagues ${ }^{145}$, made a decade ago, that characterisation of proteins associated with cfDNA may provide a rich source of information on an individual's disease, and the biology of cfDNA.

The clinical uptake of liquid biopsies will depend on the practical advantages for patients and clinicians, the infrastructure required, and its cost-effectiveness. Tissue biopsies will continue to play a key role in cancer management, particularly for the histological diagnosis and classification of cancers. At present, specialised laboratories handle CTC and ctDNA samples ${ }^{222}$, though in future hospital laboratories may carry out analysis locally if appropriate processes can be established ${ }^{223}$.

Point-of-care devices for the identification of individual hotspot mutations with clinically meaningful sensitivities are starting to be used for tissue and plasma samples $^{224,225}$. The feasibility of single molecule (third generation) sequencing of maternal plasma DNA was first demonstrated in $2015^{86}$, and subsequently it was shown that structural variants in cell line DNA can be detected ${ }^{226}$. The portability of such technologies was demonstrated by the real-time genomic surveillance in the field during the Ebola virus disease epidemic ${ }^{227}$. At present, such platforms are limited by a high error rate ${ }^{226}$, making single nucleotide variant and indel detection challenging. Another challenge is that of sequencing short DNA fragments, which 
requires optimised library preparation methods ${ }^{228}$. Sequencing capacity is also limited (currently to $\sim 150$ megabases) ${ }^{229}$, though this is likely to increase in the near future, and specific amplicons may be targeted through real-time selective sequencing ${ }^{230}$. These studies support the possibility of molecular profiling at the point of care, especially if blood plasma can be interrogated without the relatively cumbersome and time-consuming step of DNA purification ${ }^{87,231}$.

The initial approvals by the EMA and FDA for mutation detection in plasma as a companion diagnostic ${ }^{168,173,174}$, and emerging ctDNA-guided clinical trials ${ }^{209-211}$, represent key milestones towards the implementation of liquid biopsies in personalised oncology. Improving technologies are enabling an ever-wider scope for non-invasive molecular analysis of cancer, providing information that opens new avenues for genomic research and may aid in clinical decisions. In order to fully exploit the potential utility of liquid biopsies, it is essential that the biology of ctDNA be explored further. Thus far, liquid biopsies have demonstrated the potential for utility across a range of applications, and are beginning to be used for patient benefit. 


\section{Boxes}

\section{Box 1 - Clinical need for liquid biopsies in oncology}

There are multiple areas of oncology where novel diagnostics may have utility and produce clinical benefit.

- Cancer diagnosis - earlier diagnosis of cancer would enable treatment to be initiated sooner, and curative surgery may be carried out if the tumour is diagnosed at an early stage. For symptomatic patients, sensitive and specific cancer detection may speed up the time to diagnosis and treatment. At a population level, pre-symptomatic individuals may be screened for markers of disease, enabling early intervention.

- Prognosis, residual disease and risk of relapse - assessment of risk of progression is essential to select the extent or aggressiveness of treatment. After treatment with curative intent, the identification of patients with residual disease who are at high risk of relapse may be used to stratify patients to adjuvant therapy. Effective stratification would also spare low-risk patients from overtreatment.

- Treatment selection - the introduction of a wide array of novel molecularly targeted and immunotherapy agents necessitates improved tools for molecular profiling of patients and treatment stratification. At present, tumour biopsies are the standard for obtaining tumour DNA; these cannot always be obtained and their interpretation may be confounded by intratumour heterogeneity ${ }^{44-48}$, which could lead to false negative results and suboptimal therapy selection.

- Monitoring disease burden - treatment monitoring, presently performed through imaging or molecular methods, may identify response or progression, enabling clinicians and patients to adapt therapy accordingly. Current methods have limited accuracy, associated logistical burden or radiation exposure $^{50}$. The ideal monitoring assay should be repeatable serially over time, with minimal risk to patients, and should provide an accurate read-out of tumour burden. 


\section{Box 2 - Pre-analytical considerations}

In low-burden disease, or certain cancer types, the concentration of ctDNA molecules may be low and any loss of sampled material could reduce the sensitivity of molecular profiling. For quantitative applications, reproducibility of measurement is essential to achieving a robust result, and so the following pre-analytical factors should be considered:

- Samples should be collected in tubes containing an anticoagulant that is compatible with PCR, with EDTA being preferred. Plasma from heparinised blood leads to inhibition of $\mathrm{PCR}^{232}$, although some studies have been able to utilise such samples ${ }^{33}$.

- It is important that the first centrifugation of the blood is done within a few hours of the blood draw in order to remove blood cells that may lyse and release germline DNA which would dilute ctDNA ${ }^{69,233-236}$. Tubes containing fixative agents may stabilise cells and prevent lysis for several days at room temperature ${ }^{234,236-}$ ${ }^{239}$, including during shipping ${ }^{239,240}$.

- Following centrifugation, buffy coat DNA from the same tubes can be used as a source of germline DNA, although this may contain small or trace amounts of ctDNA.

- From a blood draw, plasma is preferred over serum for ctDNA analysis ${ }^{235}$. Serum also contains $c t D N A^{241}$, but blood cell lysis during the preparation of serum samples could release DNA from non-cancerous cells, which would dilute any ctDNA signal. Other body fluids or cytological specimens may be used, and may contain a higher amount or concentration of tumour DNA depending on tumour proximity (Fig. 3c).

- cfDNA extraction may be carried out with affinity-column, magnetic bead, polymer, phenol-chloroform methods, or by filtration. Different methods show variation in their ability to recover particular fragment sizes ${ }^{87,88}$, which could have implications for ctDNA detection, given the differences in size between cfDNA and ctDNA. 


\section{Figures}

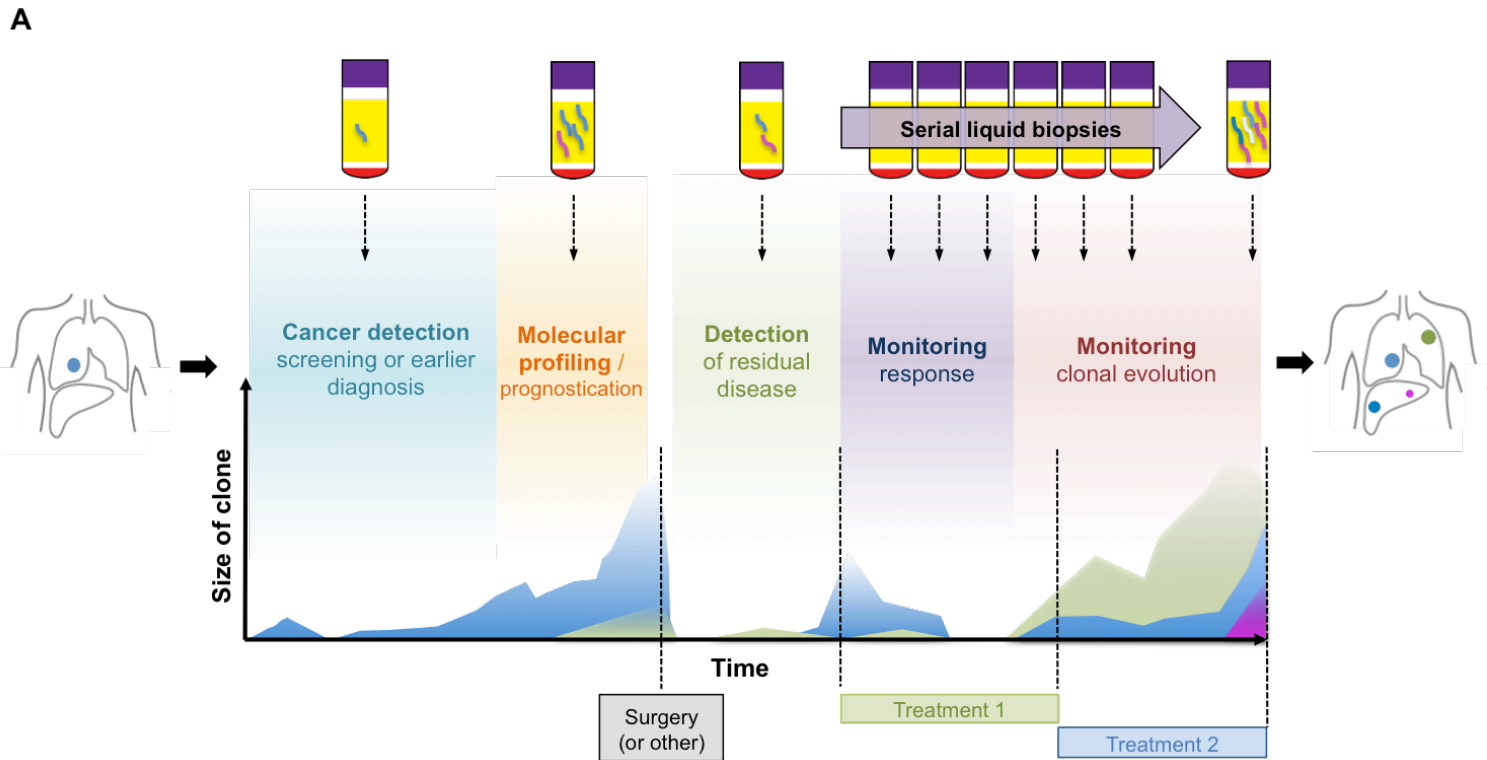

B
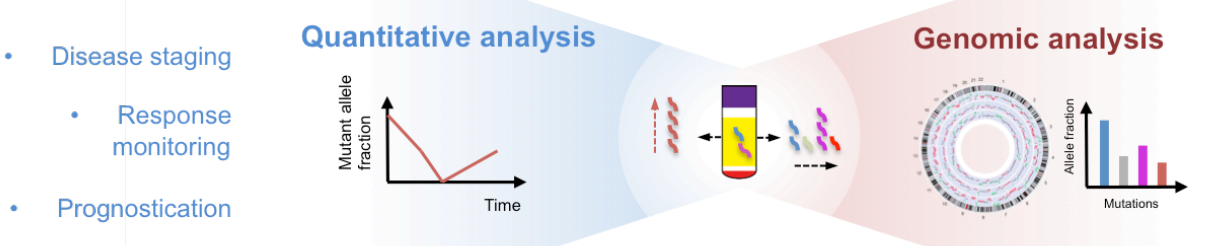

Mutation profiling

Figure 1 | Applications of ctDNA analysis during the course of disease

\section{management}

(A) A schematic time course for a hypothetical patient who undergoes surgery (or other initial treatment) has a disease relapse, and then undergoes systemic therapy. The potential applications of liquid biopsies during this patient's care are indicated. The patient starts with one single disease focus, but multiple metastases and distinct clones emerge following treatment, depicted in different colours.

(B) The information extracted from ctDNA may be classified, broadly, into quantitative information (i.e. tumour burden) or genomic information. Quantification of ctDNA at a single time point may allow disease staging and prognostication, and genomic analysis can inform selection of targeted therapies. Therefore, longitudinal analysis allows the quantitative tracking of tumour burden, such as response monitoring; and by comparing genomic profiles over time, clonal evolution may be monitored. The whole genome sequencing image was kindly provided by Dennis Lo, based on data published by Chan et al. ${ }^{38}$. 


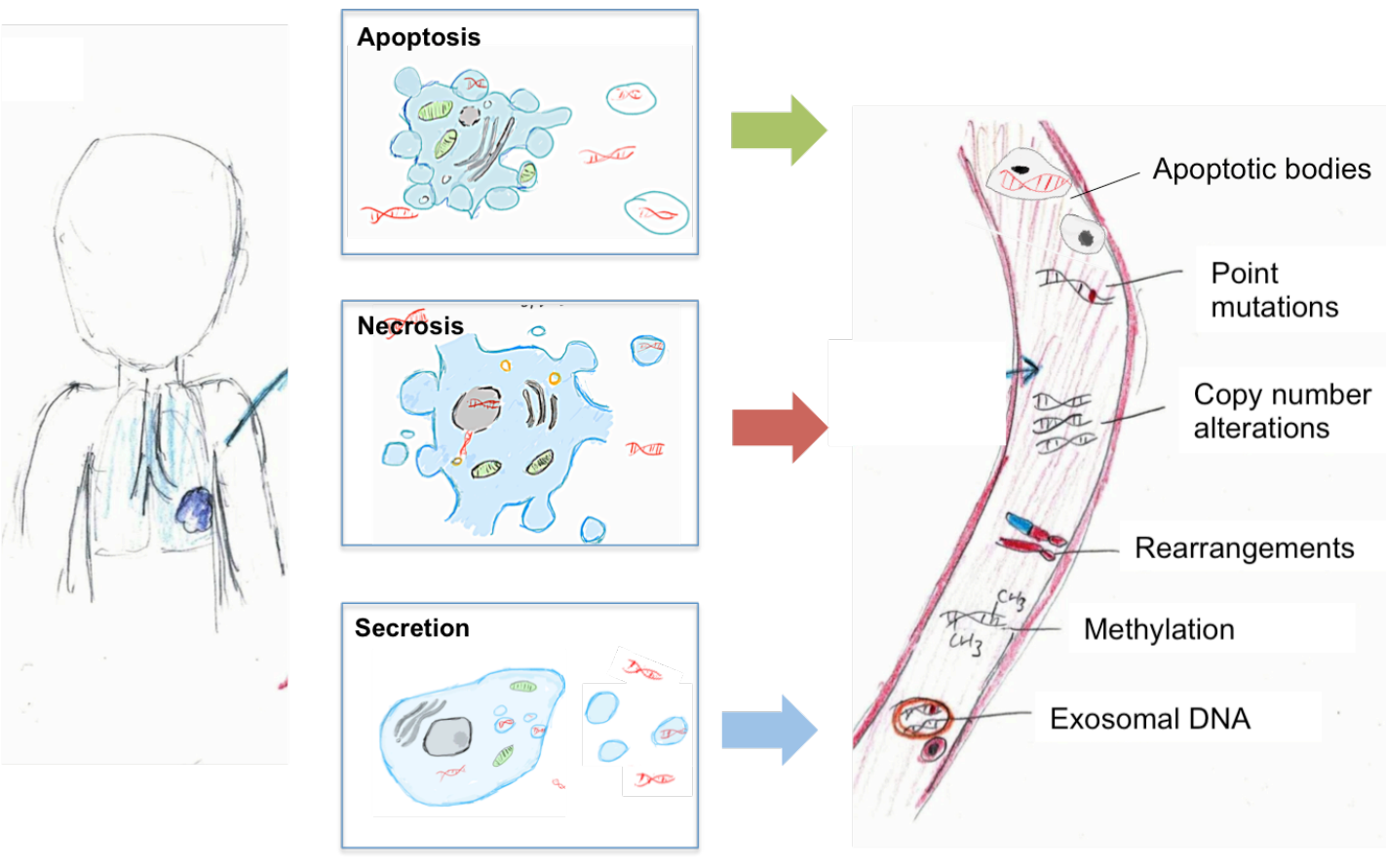

Figure 2 | Origins and spectrum of alterations in cell-free DNA

Cells release cfDNA through a combination of apoptosis, necrosis, and secretion. cfDNA can arise from cancerous cells but also from cells in the tumour microenvironment, immune cells, or other body organs. In the bloodstream cfDNA may exist as either free, or associated with extracellular vesicles such as exosomes ${ }^{2}$. Multiple classes of genetic and epigenetic alterations can be found in cell-free DNA. Adapted with permission from Schwarzenbach et al. ${ }^{215}$. 
A

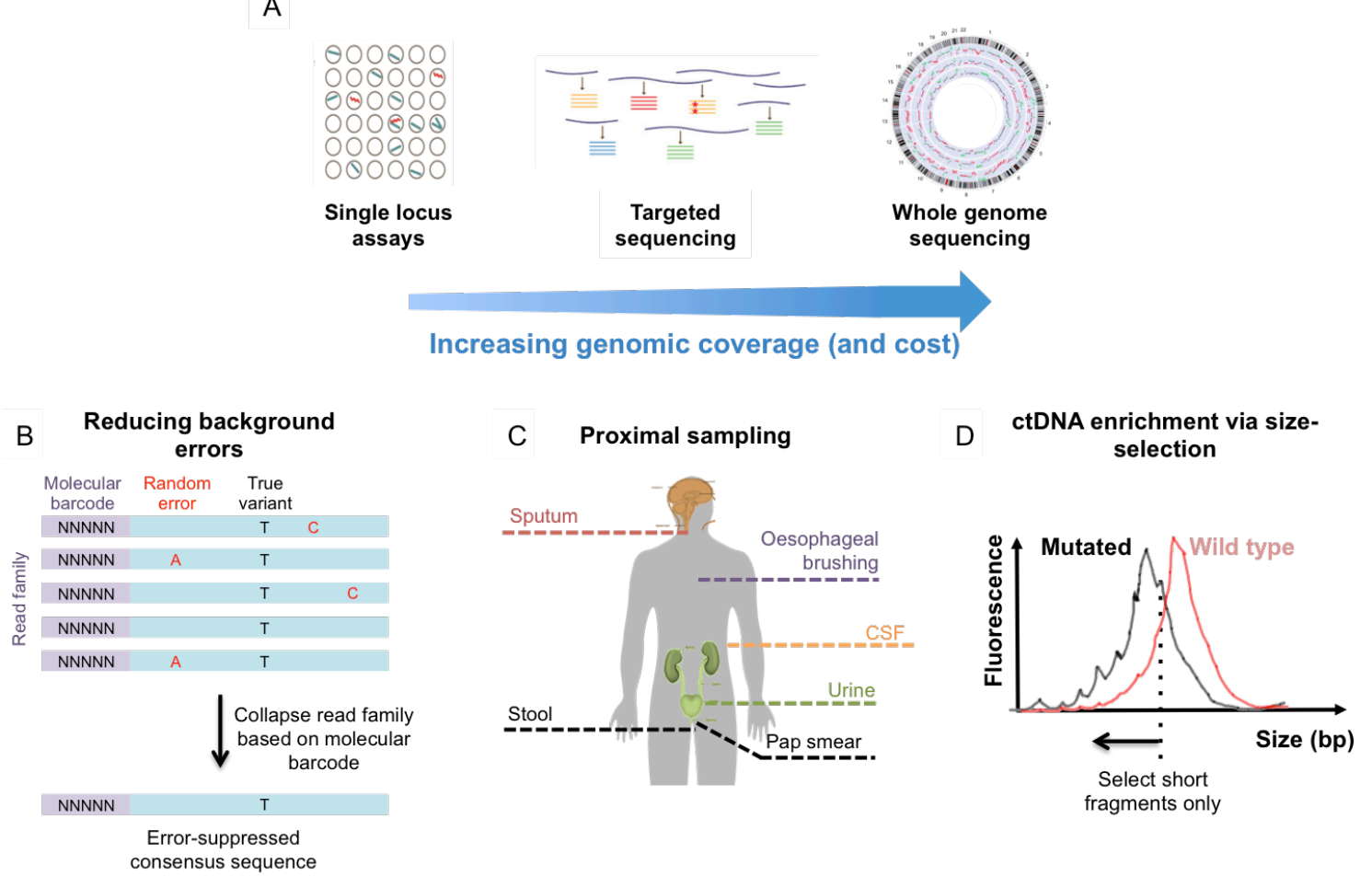

Figure 3 | Current and future paradigms for sensitive detection of ctDNA

(A) The analysis of cfDNA can range from the interrogation of individual loci, to analysing the whole genome (Table 1). Off-the-shelf dPCR assays can achieve high sensitivity with a simple workflow, but are limited by a low multiplexing capability. Targeted sequencing can allow the interrogation of multiple loci with high sensitivity, using methods that suppress background noise ${ }^{242}$. The targeted sequencing image is modified with permission from Forshew et al. ${ }^{35}$ and the whole genome sequencing image was kindly provided by Dennis Lo, based on data published by Chan et al. ${ }^{38}$.

(B) In molecular barcoding, unique molecular sequences are added to each molecule during library preparation so that sequencing reads originating from the same starting molecule can be identified. By comparing all reads from the same molecule, a single consensus sequence can be taken, which can suppress errors arising from PCR or sequencing.

(C) To improve sensitivity of analysis, for example for disease diagnosis or detection of MRD, other body fluids may be considered in combination with, or instead of, plasma. Sampling of body fluids or cytological specimens proximal to the tumour site may yield a higher concentration of DNA of tumour origin. 
(D) ctDNA has been shown to be shorter than $\operatorname{cfDNA}^{4,81,82,85}$. Thus, selection of shorter fragments experimentally or in silico may enrich for sequences of cancer origin ${ }^{82}$ and can improve sensitivity for samples with low fractions of ctDNA. 


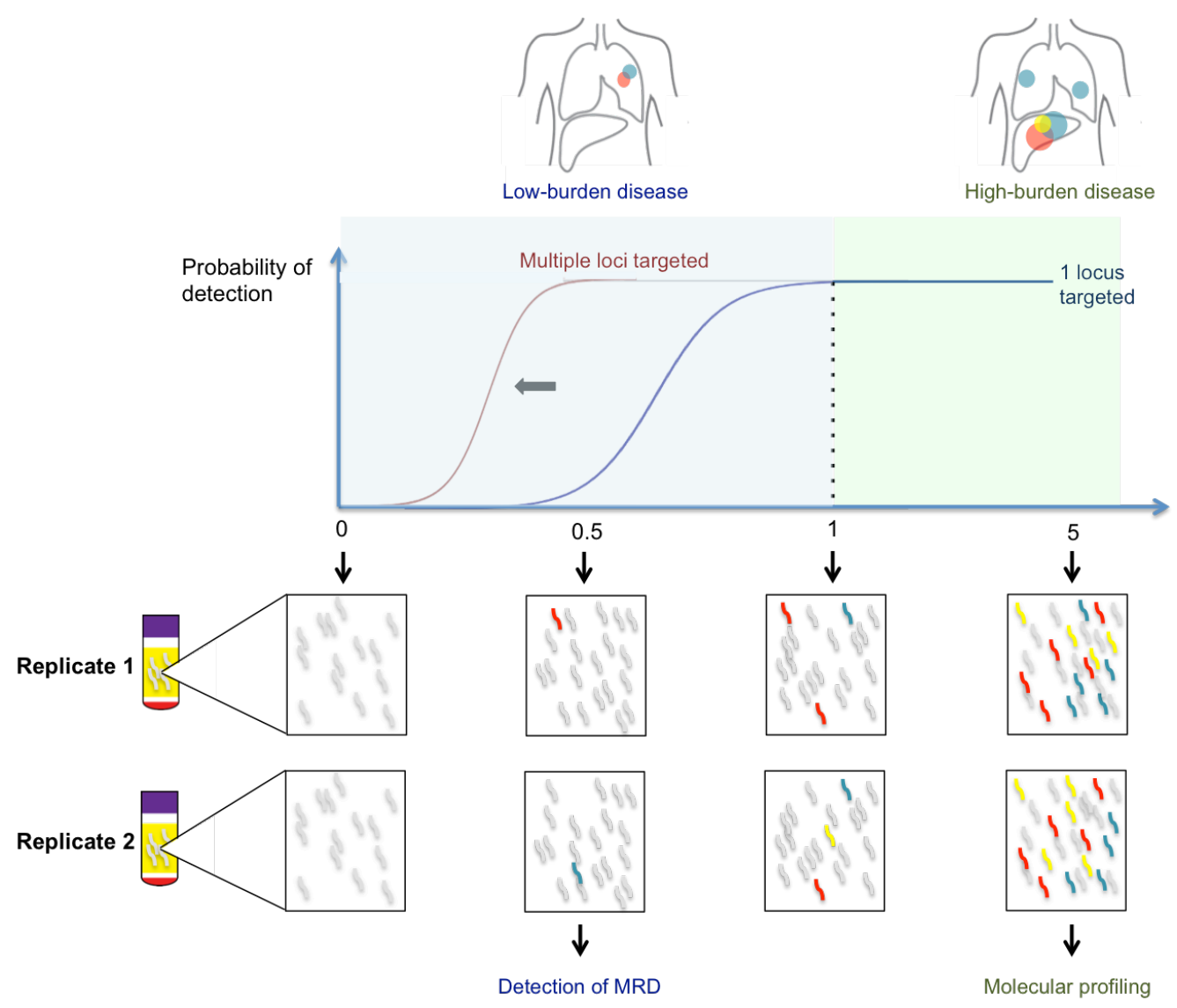

Figure 4 | Leveraging multiple mutations to detect low-burden disease and overcome sampling noise

Even with a perfectly sensitive assay, the probability of detection of ctDNA decreases as ctDNA concentration declines, as any single mutation of interest may not be present in a given volume of sample. At low ctDNA concentrations, due to sampling error, some mutations will be detected while others are missed. Sampling multiple pre-specified mutations in each reaction may improve detection of low levels of ctDNA, since every target provides an independent opportunity to test for the presence of a mutant molecule in the set of DNA molecules at that locus ${ }^{35,111}$. Sensitivity can be further improved by analysis of multiple replicates, with few molecules each, so that the mutant allele, where present in a reaction, will constitute a large fraction of the DNA template ${ }^{113}$. Boxes below the graph show hypothetical examples of sets of molecules that may be captured by each replicate in the analysis of a sample. 


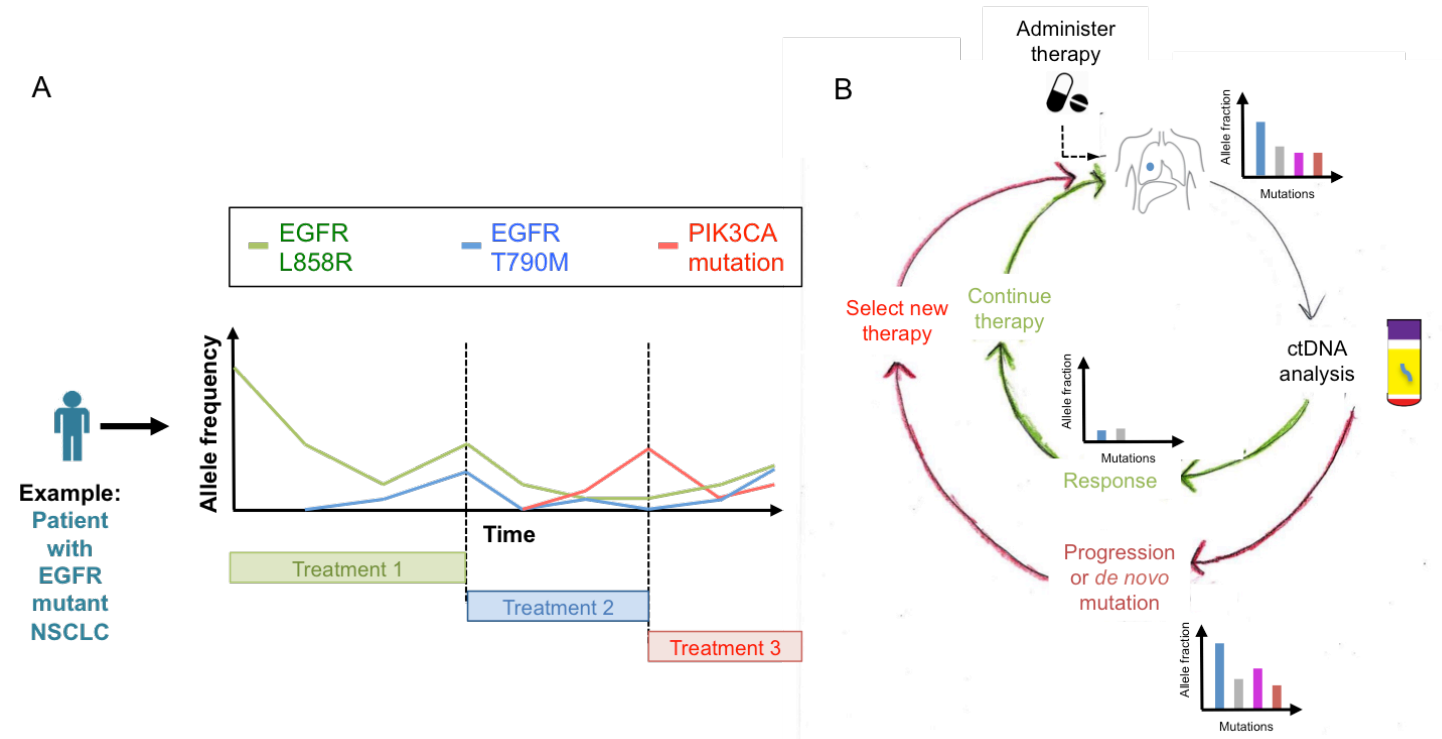

Figure 5 | Adaptive or reactive treatment paradigms using liquid biopsies

(A) During systemic anti-cancer therapy, serial liquid biopsies may identify biochemical response or progression. If progression is identified, the clinician may be able to switch therapy, or select a therapy to target arising clones carrying additional mutations that were identified by this analysis.

(B) This adaptive or reactive monitoring and treatment may continue as a loop, which would be facilitated by a fast turnaround time for ctDNA analyses, for example through the use of point-of-care diagnostics. The timeframes for this analysis can vary between hours and months; the former could allow analysis of early kinetics in response to therapy. 


\section{Tables}

Table 1 | Comparison and utility of technology platforms for ctDNA analysis

\begin{tabular}{|c|c|c|c|c|}
\hline $\begin{array}{l}\text { Scale of } \\
\text { analysis }\end{array}$ & Example technologies & $\begin{array}{l}\text { Loci } \\
\text { interrogated }\end{array}$ & $\begin{array}{l}\text { Indicative limit of } \\
\text { detection (mutant } \\
\text { allele fraction or } \\
\text { concentration) }\end{array}$ & Clinical utility \\
\hline \multirow[t]{3}{*}{$\begin{array}{l}\text { Single- } \\
\text { locus or } \\
\text { multiplexed } \\
\text { assays }\end{array}$} & 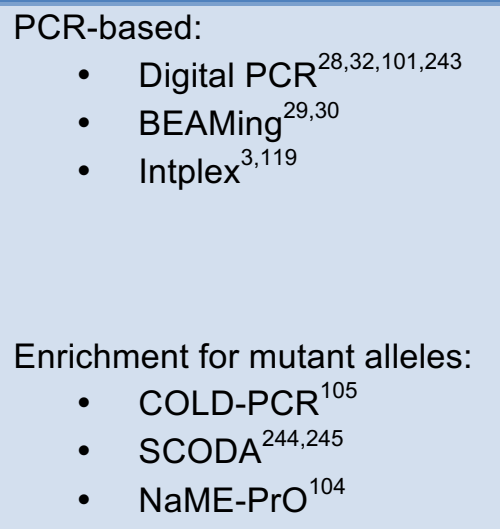 & $\begin{array}{l}\text { - } 1 \text { to } 10 \text { loci } \\
\text { - Both ctDNA and } \\
\text { cfDNA (IntPlex) }\end{array}$ & $\begin{array}{l}\text { Varies by method, } \\
\text { optimal } \\
\text { implementations can } \\
\text { reach sensitivity of } \\
0.001 \%-0.01 \% \text { or } \\
\text { individual mutant } \\
\text { copies } / \mathrm{ml}^{30,119,243,246}\end{array}$ & 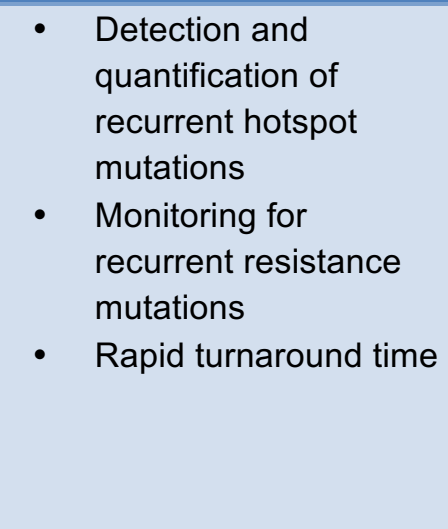 \\
\hline & $\begin{array}{l}\text { Allele-specific or ARMS-PCR } \\
\text { kits for companion diagnostics } \\
\text { (CDx): } \\
\text { - cobas EGFR }{ }^{168}\end{array}$ & $\begin{array}{l}7 \text { mutation assays } \\
\text { covering multiple } \\
\text { variants }\end{array}$ & $\begin{array}{l}\text { Limit of detection } \\
\text { ( } \geq 95 \% \text { sensitivity): } \\
25-100 \text { copies } / \mathrm{ml}^{168}\end{array}$ & $\begin{array}{l}\text { Approved for in vitro } \\
\text { diagnostic (IVD) use: } \\
\text { - FDA-approved }\end{array}$ \\
\hline & - therascreen EGFR ${ }^{166}$ & $\begin{array}{l}3 \text { mutation assays } \\
\text { covering multiple } \\
\text { variants }\end{array}$ & $\begin{array}{l}\text { Median } 1.42 \% \\
\text { (range } 0.05 \% \text { - } \\
12.47 \% \text { for different } \\
\text { variants) }\end{array}$ & - CE-marked \\
\hline $\begin{array}{l}\text { Targeted } \\
\text { sequencing } \\
\text { approaches }\end{array}$ & 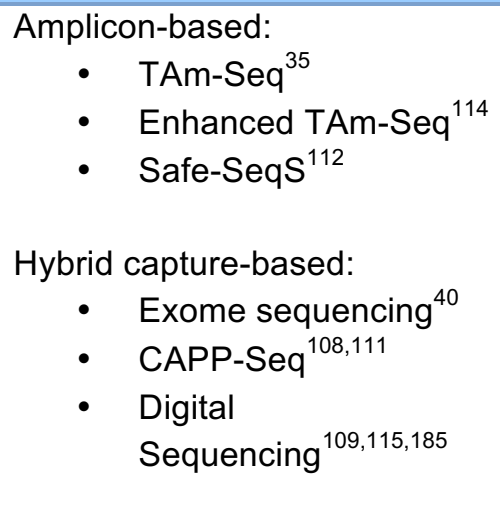 & $\begin{array}{l}10 \text { kilobases to } 50 \\
\text { megabases }\end{array}$ & $\begin{array}{l}<0.01 \%-0.5 \% \text { for } \\
\text { purpose-built } \\
\text { panels } \\
14,109,111,112,176,1 \\
1 \% \text { for off-the-shelf } \\
\text { multiplexed } \\
\text { panels }^{46,110} \\
5 \% \text { for exome } \\
\text { sequencing }^{40}\end{array}$ & $\begin{array}{l}\text { - Profiling gene panels } \\
\text { - } \text { Monitoring for de novo } \\
\text { resistance mutations. } \\
\text { Monitoring clonal } \\
\text { evolution in response } \\
\text { to therapy. } \\
\text { - Sensitivity for disease } \\
\text { burden can be } \\
\text { increased by testing } \\
\text { multiple loci in parallel } \\
\text { (Fig. 4) }\end{array}$ \\
\hline $\begin{array}{l}\text { Genome- } \\
\text { wide }\end{array}$ & $\begin{array}{l}\text { WGS: } \\
\text { • } \text { Plasma-Seq }^{39} \\
\text { - } \text { PARE }^{197} \\
\text { Amplicon-based: } \\
\text { - FAST-SeqS }{ }^{184} \\
\text { - } \text { mFAST-SeqS }^{116}\end{array}$ & $\begin{array}{l}3.2 \text { gigabases } \\
\text { (whole genome) } \\
21.6 \text { unique } \\
\text { kilobases of LINE- } \\
1^{184}\end{array}$ & $5 \%-10 \%{ }^{39}$ & 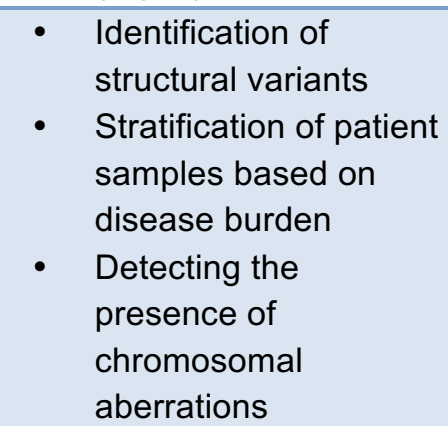 \\
\hline
\end{tabular}


Table 1 | Abbreviations used: BEAMing, beads, emulsion, amplification, and magnetics; CAPP-Seq, cancer personalized profiling by deep sequencing; COLDPCR, co-amplification at lower denaturation temperature PCR; FAST-SeqS, fast aneuploidy screening test-sequencing system; LINE-1, long interspersed nucleotide element-1; mFAST-SeqS, modified fast aneuploidy screening test-sequencing system; NaME-PrO, nuclease-assisted minor-allele enrichment with probe-overlap; PARE, personalized analysis of rearranged ends; SCODA, synchronous coefficient of drag alteration; TAm-Seq, tagged amplicon deep sequencing 


\section{References}

1. Mandel, P. \& Métais, P. Les acides nucléiques du plasma sanguin chez l'homme. C. $R$. Seances Soc. Biol. Fil. 142, 241-243 (1948).

2. Thierry, A. R., El Messaoudi, S., Gahan, P. B., Anker, P. \& Stroun, M. Origins, structures, and functions of circulating DNA in oncology. Cancer Metastasis Rev. 35, 347-376 (2016).

3. Mouliere, F., El Messaoudi, S., Pang, D., Dritschilo, A. \& Thierry, A. R. Multi-marker analysis of circulating cell-free DNA toward personalized medicine for colorectal cancer. Mol. Oncol. 8, 927-941 (2014).

4. Mouliere, F. et al. High Fragmentation Characterizes Tumour-Derived Circulating DNA. PLoS One 6, e23418 (2011).

5. Leon, S. A., Shapiro, B., Sklaroff, D. M. \& Yaros, M. J. Free DNA in the serum of cancer patients and the effect of therapy. Cancer Res. 37, 646-650 (1977).

6. Rodrigues Filho, E. M. et al. Elevated Cell-Free Plasma DNA Level as an Independent Predictor of Mortality in Patients with Severe Traumatic Brain Injury. J. Neurotrauma 31, 1639-1646 (2014).

7. Tsai, N.-W. et al. The value of serial plasma nuclear and mitochondrial DNA levels in patients with acute ischemic stroke. Clin. Chim. Acta 412, 476-479 (2011).

8. Breitbach, S., Sterzing, B., Magallanes, C., Tug, S. \& Simon, P. Direct measurement of cellfree DNA from serially collected capillary plasma during incremental exercise. J. Appl. Physiol. 117, 119-130 (2014).

9. De Vlaminck, I. et al. Circulating cell-free DNA enables noninvasive diagnosis of heart transplant rejection. Sci. Transl. Med. 6, 241 ra77 (2014).

10. De Vlaminck, I. et al. Noninvasive monitoring of infection and rejection after lung transplantation. Proc. Natl. Acad. Sci. 112, 13336-41 (2015).

11. Lo, Y. M. D. et al. Presence of fetal DNA in maternal plasma and serum. Lancet 350, 485487 (1997).

12. Hyett, J. A. et al. Reduction in diagnostic and therapeutic interventions by non-invasive determination of fetal sex in early pregnancy. Prenat. Diagn. 25, 1111-1116 (2005).

13. Saito, H., Sekizawa, A., Morimoto, T., Suzuki, M. \& Yanaihara, T. Prenatal DNA diagnosis of a single-gene disorder from maternal plasma. Lancet 356, 1170 (2000).

14. Lo, Y. M. D. et al. Digital PCR for the molecular detection of fetal chromosomal aneuploidy. Proc. Natl. Acad. Sci. 104, 13116-13121 (2007).

15. Allyse, M. et al. Non-invasive prenatal testing: A review of international implementation and challenges. Int. J. Womens. Health 7, 113-126 (2015).

16. Hill, M. et al. Evaluation of non-invasive prenatal testing (NIPT) for aneuploidy in an NHS setting: a reliable accurate prenatal non-invasive diagnosis (RAPID) protocol. BMC Pregnancy Childbirth 14, 229 (2014).

17. Stroun, M. et al. Neoplastic Characteristics of the DNA Found in the Plasma of Cancer Patients. Oncology 46, 318-322 (1989).

18. Sidransky, D. et al. Identification of p53 gene mutations in bladder cancers and urine samples. Science 252, 706-709 (1991).

19. Sidransky, D. et al. Identification of ras oncogene mutations in the stool of patients with curable colorectal tumors. Science 256, 102-5 (1992).

20. Caldas, C. et al. Detection of K-ras mutations in the stool of patients with pancreatic adenocarcinoma and pancreatic ductal hyperplasia. Cancer Res. 54, 3568-3573 (1994).

21. Mao, L., Hruban, R. H., Boyle, J. O., Tockman, M. \& Sidransky, D. Detection of Oncogene Mutations in Sputum Precedes Diagnosis of Lung Cancer. Cancer Res. 54, 1634-1637 (1994).

22. Takeda, S., Ichii, S. \& Nakamura, Y. Detection of K-ras mutation in sputum by mutant-allelespecific amplification (MASA). Hum. Mutat. 2, 112-117 (1993).

23. Sorenson, G. D. et al. Soluble Normal and Mutated Dna-Sequences From Single-Copy Genes in Human Blood. Cancer Epidemiol. Biomarkers Prev. 3, 67-71 (1994).

24. Swisher, E. M. et al. Tumor-specific p53 sequences in blood and peritoneal fluid of women with epithelial ovarian cancer. Am. J. Obstet. Gynecol. 193, 662-667 (2005).

25. Kimura, H. et al. Detection of Epidermal Growth Factor Receptor Mutations in Serum as a Predictor of the Response to Gefitinib in Patients with Non-Small-Cell Lung Cancer. Clin Cancer Res 12, 3915-3921 (2006).

26. Sozzi, G., Musso, K., Ratcliffe, C., Goldstraw, P. \& Pierotti, M. A. Detection of Microsatellite Alterations in Plasma DNA of Non-Small Cell Lung Cancer Patients: A Prospect for Early Diagnosis. Clin Cancer Res 5, 2689-2692 (1999).

27. Lecomte, T. et al. Detection of free-circulating tumor-associated DNA in plasma of colorectal cancer patients and its association with prognosis. Int. J. Cancer 100, 542-548 (2002).

28. Vogelstein, B. \& Kinzler, K. W. Digital PCR. Proc. Natl. Acad. Sci. 96, 9236-9241 (1999). 
29. Dressman, D., Yan, H., Traverso, G., Kinzler, K. W. \& Vogelstein, B. Transforming single DNA molecules into fluorescent magnetic particles for detection and enumeration of genetic variations. Proc. Natl. Acad. Sci. U. S. A. 100, 8817-8822 (2003).

30. Diehl, F. et al. Detection and quantification of mutations in the plasma of patients with colorectal tumors. Proc. Natl. Acad. Sci. 102, 16368-16373 (2005).

31. Diehl, F. et al. Circulating mutant DNA to assess tumor dynamics. Nat. Med. 14, 985-990 (2008).

32. Yung, T. K. F. et al. Single-molecule detection of epidermal growth factor receptor mutations in plasma by microfluidics digital PCR in non-small cell lung cancer patients. Clin. Cancer Res. 15, 2076-2084 (2009).

33. Kuang, Y. et al. Noninvasive Detection of EGFR T790M in Gefitinib or Erlotinib Resistant Non-Small Cell Lung Cancer. Clin. Cancer Res. 15, 2630-2636 (2009).

34. Qian, X. et al. Circulating cell-free DNA has a high degree of specificity to detect exon 19 deletions and the single-point substitution mutation L858R in non-small cell lung cancer. Oncotarget 7, 29154-29165 (2016).

35. Forshew, T. et al. Noninvasive Identification and Monitoring of Cancer Mutations by Targeted Deep Sequencing of Plasma DNA. Sci. Transl. Med. 4, 136ra68-136ra68 (2012).

36. Dawson, S.-J. et al. Analysis of circulating tumor DNA to monitor metastatic breast cancer. $N$. Engl. J. Med. 368, 1199-209 (2013).

37. Leary, R. J. et al. Detection of Chromosomal Alterations in the Circulation of Cancer Patients with Whole-Genome Sequencing. Sci. Transl. Med. 4, 162ra154 (2012).

38. Chan, K. C. A. et al. Cancer Genome Scanning in Plasma: Detection of Tumor-Associated Copy Number Aberrations, Single-Nucleotide Variants, and Tumoral Heterogeneity by Massively Parallel Sequencing. Clin. Chem. 59, 211-224 (2013).

39. Heitzer, E. et al. Tumor-associated copy number changes in the circulation of patients with prostate cancer identified through whole-genome sequencing. Genome Med. 5, 30 (2013).

40. Murtaza, M. et al. Non-invasive analysis of acquired resistance to cancer therapy by sequencing of plasma DNA. Nature 497, 108-12 (2013).

41. Overman, M. J. et al. Use of research biopsies in clinical trials: Are risks and benefits adequately discussed? J. Clin. Oncol. 31, 17-22 (2013).

42. VanderLaan, P. A. et al. Success and failure rates of tumor genotyping techniques in routine pathological samples with non-small-cell lung cancer. Lung Cancer 84, 39-44 (2014).

43. Ellis, P. M. et al. Dacomitinib compared with placebo in pretreated patients with advanced or metastatic non-small-cell lung cancer (NCIC CTG BR.26): A double-blind, randomised, phase 3 trial. Lancet Oncol. 15, 1379-1388 (2014).

44. Popper, H. H. Commentary on tumor heterogeneity. Transl. Lung Cancer Res. 5, 433-435 (2016).

45. De Mattos-Arruda, L. et al. Capturing Intra-Tumor Genetic Heterogeneity by De Novo Mutation Profiling of Circulating Cell-Free Tumor DNA: A Proof-of-Principle. Ann. Oncol. 25, 1729-1735 (2014).

46. Lebofsky, R. et al. Circulating tumor DNA as a non-invasive substitute to metastasis biopsy for tumor genotyping and personalized medicine in a prospective trial across all tumor types. Mol. Oncol. 9, 783-790 (2015).

47. Jamal-Hanjani, M. et al. Detection of Ubiquitous and Heterogeneous Mutations in Cell-Free DNA from Patients with Early-Stage Non-Small-Cell Lung Cancer. Ann. Oncol. 27, 862-7 (2016).

48. Murtaza, M. et al. Multifocal clonal evolution characterized using circulating tumour DNA in a case of metastatic breast cancer. Nat. Commun. 6, 8760 (2015).

49. Duffy, M. J. Serum tumor markers in breast cancer: Are they of clinical value? Clin. Chem. 52, 345-351 (2006).

50. Fazel, R. et al. Exposure to Low-Dose Ionizing Radiation from Medical Imaging Procedures. N. Engl. J. Med. 361, 849-857 (2009).

51. Thakur, B. K. et al. Double-stranded DNA in exosomes: a novel biomarker in cancer detection. Cell Res. 24, 766-9 (2014).

52. Kahlert, C. et al. Identification of Double-stranded Genomic DNA Spanning All Chromosomes with Mutated KRAS and p53 DNA in the Serum Exosomes of Patients with Pancreatic Cancer. J. Biol. Chem. 289, 3869-3875 (2014).

53. Jahr, S. et al. DNA Fragments in the Blood Plasma of Cancer Patients: Quantitations and Evidence for Their Origin from Apoptotic and Necrotic Cells. Cancer Res. 61, 1659-1665 (2001).

54. Stroun, M., Lyautey, J., Lederrey, C., Olson-Sand, A. \& Anker, P. About the possible origin and mechanism of circulating DNA: Apoptosis and active DNA release. Clin. Chim. Acta 313, 139-142 (2001).

55. Botezatu, I. et al. Genetic Analysis of DNA Excreted in Urine: A New Approach for Detecting 
Specific Genomic DNA Sequences from Cells Dying in an Organism. Clin. Chem. 46, 1078$1084(2000)$.

56. Chan, K. C. A., Leung, S. F., Yeung, S. W., Chan, A. T. C. \& Lo, Y. M. D. Quantitative analysis of the transrenal excretion of circulating EBV DNA in nasopharyngeal carcinoma patients. Clin. Cancer Res. 14, 4809 (2008).

57. Birkenkamp-Demtröder, K. et al. Genomic Alterations in Liquid Biopsies from Patients with Bladder Cancer. Eur. Urol. 70, 75-82 (2016).

58. Reckamp, K. L. et al. A Highly Sensitive and Quantitative Test Platform for Detection of NSCLC EGFR Mutations in Urine and Plasma. J. Thorac. Oncol. 11, 1690-1700 (2016).

59. Su, Y.-H. et al. Human Urine Contains Small, 150 to 250 Nucleotide- Sized, Soluble DNA Derived from the Circulation and May Be Useful in the Detection of Colorectal Cancer. J. Mol. Diagnostics 6, 101-7 (2004).

60. Wang, Y. et al. Detection of tumor-derived DNA in cerebrospinal fluid of patients with primary tumors of the brain and spinal cord. Proc. Natl. Acad. Sci. 112, 9704-9 (2015).

61. Pan, W., Gu, W., Nagpal, S., Gephart, M. H. \& Quake, S. R. Brain Tumor Mutations Detected in Cerebral Spinal Fluid. Clin. Chem. 61, 514-522 (2015).

62. De Mattos-Arruda, L. et al. Cerebrospinal fluid-derived circulating tumour DNA better represents the genomic alterations of brain tumours than plasma. Nat. Commun. 6, 8839 (2015).

63. Rhodes, C. H., Honsinger, C. \& Sorenson, G. D. PCR-detection of tumor-derived p53 DNA in cerebrospinal fluid. Am. J. Clin. Pathol. 103, 404-408 (1995).

64. Sriram, K. B. et al. Pleural fluid cell-free DNA integrity index to identify cytologically negative malignant pleural effusions including mesotheliomas. BMC Cancer 12, 428 (2012).

65. Mithani, S. K. et al. Mitochondrial resequencing arrays detect tumor-specific mutations in salivary rinses of patients with head and neck cancer. Clin. Cancer Res. 13, 7335-7340 (2007).

66. Snyder, M. W., Kircher, M., Hill, A. J., Daza, R. M. \& Shendure, J. Cell-free DNA Comprises an In Vivo Nucleosome Footprint that Informs Its Tissues-Of-Origin. Cell 164, 57-68 (2016).

67. Lehmann-Werman, R. et al. Identification of tissue-specific cell death using methylation patterns of circulating DNA. Proc. Natl. Acad. Sci. 113, E1826-34 (2016).

68. Sun, K. et al. Plasma DNA tissue mapping by genome-wide methylation sequencing for noninvasive prenatal, cancer, and transplantation assessments. Proc. Natl. Acad. Sci. 112, E5503-E5512 (2015).

69. Lui, Y. Y. N. et al. Predominant hematopoietic origin of cell-free DNA in plasma and serum after sex-mismatched bone marrow transplantation. Clin. Chem. 48, 421-427 (2002).

70. Tug, S. et al. Exercise-induced increases in cell free DNA in human plasma originate predominantly from cells of the haematopoietic lineage. Exerc. Immunol. Rev. 21, 164-173 (2015).

71. To, E. W. H. et al. Rapid Clearance of Plasma Epstein-Barr Virus DNA After Surgical Treatment of Nasopharyngeal Carcinoma. Clin. Cancer Res. 9, 3254-3259 (2003).

72. Lo, Y. M. et al. Rapid clearance of fetal DNA from maternal plasma. Am. J. Hum. Genet. 64, 218-24 (1999).

73. Wang, Y., Chen, M., Xiao, N. \& Liu, H. Evaluation and comparison of in vitro degradation kinetics of DNA in serum, urine and saliva: A qualitative Study. Gene 590, 142-148 (2016).

74. Tamkovich, S. N. et al. Circulating DNA and DNase activity in human blood. Ann. N. Y. Acad. Sci. 1075, 191-196 (2006).

75. Tsui, N. B. Y. et al. High Resolution Size Analysis of Fetal DNA in the Urine of Pregnant Women by Paired-End Massively Parallel Sequencing. PLoS One 7, e48319 (2012).

76. Chused, T. M., Steinberg, a D. \& Talal, N. The clearance and localization of nucleic acids by New Zealand and normal mice. Clin. Exp. Immunol. 12, 465-76 (1972).

77. Giacona, M. B. et al. Cell-free DNA in human blood plasma: length measurements in patients with pancreatic cancer and healthy controls. Pancreas 17, 89-97 (1998).

78. Lo, Y. M. D. et al. Maternal plasma DNA sequencing reveals the genome-wide genetic and mutational profile of the fetus. Sci. Transl. Med. 2, 61 ra91 (2010).

79. Thierry, A. R. et al. Origin and quantification of circulating DNA in mice with human colorectal cancer xenografts. Nucleic Acids Res. 38, 6159-6175 (2010).

80. Nadano, D., Yasuda, T. \& Kishi, K. Measurement of deoxyribonuclease I activity in human tissues and body fluids by a single radial enzyme-diffusion method. Clin. Chem. 39, 448-452 (1993).

81. Mouliere, F. et al. Circulating Cell-Free DNA from Colorectal Cancer Patients May Reveal High KRAS or BRAF Mutation Load. Transl. Oncol. 6, 319-328 (2013).

82. Underhill, H. R. et al. Fragment Length of Circulating Tumor DNA. PLOS Genet 12, 426-37 (2016).

83. Gorges, T. M. et al. Cancer therapy monitoring in xenografts by quantitative analysis of 
circulating tumor DNA. Biomarkers 17, 498-506 (2012).

84. Zheng, Y. W. L. et al. Nonhematopoietically derived DNA is shorter than hematopoietically derived DNA in plasma: A transplantation model. Clin. Chem. 58, 549-558 (2012).

85. Jiang, P. \& Lo, Y. M. D. The Long and Short of Circulating Cell-Free DNA and the Ins and Outs of Molecular Diagnostics. Trends Genet. 32, 360-371 (2016).

86. Cheng, $\mathrm{S}$. H. et al. Noninvasive prenatal testing by nanopore sequencing of maternal plasma DNA: Feasibility assessment. Clin. Chem. 61, 1305-1306 (2015).

87. Breitbach, S. et al. Direct Quantification of Cell-Free, Circulating DNA from Unpurified Plasma. PLoS One 9, e87838 (2014).

88. Beránek, M. et al. Carrier molecules and extraction of circulating tumor DNA for next generation sequencing in colorectal cancer. Acta Medica (Hradec Kralove) 59, 54-58 (2016).

89. Gansauge, M.-T. \& Meyer, M. Single-stranded DNA library preparation for the sequencing of ancient or damaged DNA. Nat. Protoc. 8, 737-48 (2013).

90. Burnham, P. et al. Single-stranded DNA library preparation uncovers the origin and diversity of ultrashort cell-free DNA in plasma. Sci. Rep. 6, 27859 (2016).

91. Kostyuk, S. V. et al. Fragments of cell-free DNA increase transcription in human mesenchymal stem cells, activate TLR-dependent signal pathway, and suppress apoptosis. Biochem. Suppl. Ser. B Biomed. Chem. 6, 68-74 (2012).

92. Nishimoto, S. et al. Obesity-induced DNA released from adipocytes stimulates chronic adipose tissue inflammation and insulin resistance. Sci. Adv. 2, e1501332 (2016).

93. Dvořáková, M. et al. DNA released by leukemic cells contributes to the disruption of the bone marrow microenvironment. Oncogene 32, 5201-5209 (2013).

94. Porath, J. \& GARTLER, S. M. Cellular Uptake of Deoxyribonucleic Acid by Human Tissue Culture Cells. Nature 184, 1505-1506 (1959).

95. García-Olmo, D. C. et al. Cell-free nucleic acids circulating in the plasma of colorectal cancer patients induce the oncogenic transformation of susceptible cultured cells. Cancer Res. 70, 560-567 (2010).

96. Ju, Y. S. et al. Frequent somatic transfer of mitochondrial DNA into the nuclear genome of human cancer cells. Genome Res. 25, 814-824 (2015).

97. Wu, D. Y., Ugozzoli, L., Pal, B. K. \& Wallace, R. B. Allele-specific enzymatic amplification of beta-globin genomic DNA for diagnosis of sickle cell anemia. Proc. Natl. Acad. Sci. U. S. A. 86, 2757-2760 (1989).

98. Newton, C. R. et al. Analysis of any point mutation in DNA. The amplification refractory mutation system (ARMS). Nucleic Acids Res. 17, 2503-2516 (1989).

99. Normanno, N., Denis, M. G., Thress, K. S., Ratcliffe, M. \& Reck, M. Guide to detecting epidermal growth factor receptor (EGFR) mutations in ctDNA of patients with advanced nonsmall-cell lung cancer. Oncotarget (2016). doi:10.18632/oncotarget.13915

100. Schiavon, G. et al. Analysis of ESR1 mutation in circulating tumor DNA demonstrates evolution during therapy for metastatic breast cancer. Sci. Transl. Med. 7, 313ra182313ra182 (2015).

101. Taly, V. et al. Multiplex picodroplet digital PCR to detect KRAS mutations in circulating DNA from the plasma of colorectal cancer patients. Clin. Chem. 59, 1722-1731 (2013).

102. Sacher, A. G. et al. Prospective Validation of Rapid Plasma Genotyping for the Detection of EGFR and KRAS Mutations in Advanced Lung Cancer. JAMA Oncol. 2, 1014-22 (2016).

103. Mosko, M. J. et al. Ultrasensitive Detection of Multiplexed Somatic Mutations Using MALDITOF Mass Spectrometry. J. Mol. diagnostics 18, 23-31 (2016).

104. Song, C. et al. Elimination of unaltered DNA in mixed clinical samples via nuclease-assisted minor-allele enrichment. Nucleic Acids Res. 44, 1-11 (2016).

105. Li, J. et al. Replacing PCR with COLD-PCR enriches variant DNA sequences and redefines the sensitivity of genetic testing. Nat. Med. 14, 579-584 (2008).

106. Guha, M., Castellanos-Rizaldos, E., Liu, P., Mamon, H. \& Makrigiorgos, G. M. Differential strand separation at critical temperature: A minimally disruptive enrichment method for lowabundance unknown DNA mutations. Nucleic Acids Res. 41, e50 (2013).

107. Gadgeel, S. et al. Assessment of EGFR mutations in matched urine, plasma and tumor tissue in NSCLC patients treated with rociletinib (CO-1686). in Proceedings of the AACRNCI-EORTC International Conference: Molecular Targets and Cancer Therapeutics (AACR; Mol Cancer Ther, 2015).

108. Newman, A. M. et al. An ultrasensitive method for quantitating circulating tumor DNA with broad patient coverage. Nat. Med. 20, 548-54 (2014).

109. Lanman, R. B. et al. Analytical and clinical validation of a digital sequencing panel for quantitative, highly accurate evaluation of cell-free circulating tumor DNA. PLOS One 10, e0140712 (2015).

110. Frenel, J.-S. et al. Serial Next Generation Sequencing of Circulating Cell Free DNA Evaluating Tumour Clone Response To Molecularly Targeted Drug Administration. Clin. 
Cancer Res. 21, 4586-96 (2015).

111. Newman, A. M. et al. Integrated digital error suppression for improved detection of circulating tumor DNA. Nat Biotechnol 34, 547-55 (2016).

112. Kinde, I., Wu, J., Papadopoulos, N., Kinzler, K. W. \& Vogelstein, B. Detection and quantification of rare mutations with massively parallel sequencing. Proc. Natl. Acad. Sci. 108, 9530-9535 (2011).

113. Rosenfeld, N., Forshew, T., Marass, F. \& Murtaza, M. Patent WO2016009224 A1 - A method for detecting a genetic variant. (2016).

114. Gale, D. et al. Abstract 3639: Analytical performance and validation of an enhanced TAmSeq circulating tumor DNA sequencing assay. Cancer Res. 76, 3639 (2016).

115. Schwaederle, M. C. et al. Detection rate of actionable mutations in diverse cancers using a biopsy-free (blood) circulating tumor DNA assay. Oncotarget 33, 11004 (2015).

116. Belic, J. et al. Rapid Identification of Plasma DNA Samples with Increased ctDNA Levels by a Modified FAST-SeqS Approach. Clin. Chem. 61, 838-849 (2015).

117. Kirkizlar, E. et al. Detection of Clonal and Subclonal Copy-Number Variants in Cell-Free DNA from Patients with Breast Cancer Using a Massively Multiplexed PCR Methodology. Transl. Oncol. 8, 407-416 (2015).

118. Parkinson, C. A. et al. Exploratory analysis of TP53 mutations in circulating tumour DNA as biomarkers of treatment response for patients with relapsed high-grade serous ovarian carcinoma: a retrospective study. PLoS Med. 13, e1002198 (2016).

119. Thierry, A. R. et al. Clinical validation of the detection of KRAS and BRAF mutations from circulating tumor DNA. Nat. Med. 20, 430-435 (2014).

120. Kamat, A. A. et al. Circulating cell-free DNA: A novel biomarker for response to therapy in ovarian carcinoma. Cancer Biology \& Therapy 5, 1369-1374 (2006).

121. Bettegowda, C. et al. Detection of Circulating Tumor DNA in Early- and Late-Stage Human Malignancies. Sci. Transl. Med. 6, 224ra24 (2014).

122. Nygaard, A. D., Garm Spindler, K.-L., Pallisgaard, N., Andersen, R. F. \& Jakobsen, A. The prognostic value of KRAS mutated plasma DNA in advanced non-small cell lung cancer. Lung Cancer 79, 312-7 (2013).

123. Wang, S. et al. Potential clinical significance of a plasma-based KRAS mutation analysis in patients with advanced non-small cell lung cancer. Clin. Cancer Res. 16, 1324-1330 (2010).

124. Gautschi, O. et al. Origin and prognostic value of circulating KRAS mutations in lung cancer patients. Cancer Lett. 254, 265-273 (2007).

125. Santiago-Walker, A. et al. Correlation of BRAF mutation status in circulating-free DNA and tumor and association with clinical outcome across four BRAFi and MEKi clinical trials. Clin. Cancer Res. 22, 567-74 (2016).

126. Gray, E. S. et al. Circulating tumor DNA to monitor treatment response and detect acquired resistance in patients with metastatic melanoma. Oncotarget 6, 42008-18 (2015).

127. Lipson, E. J. et al. Circulating tumor DNA analysis as a real-time method for monitoring tumor burden in melanoma patients undergoing treatment with immune checkpoint blockade. J. Immunother. Cancer 2, 42 (2014).

128. Oshiro, C. et al. PIK3CA mutations in serum DNA are predictive of recurrence in primary breast cancer patients. Breast Cancer Res. Treat. 150, 299-307 (2015).

129. Scherer, F. et al. Distinct biological subtypes and patterns of genome evolution in lymphoma revealed by circulating tumor DNA. Sci. Transl. Med. 8, 364ra155 (2016).

130. Office for National Statistics. Cancer survival by stage at diagnosis for England (experimental statistics): Adults diagnosed 2012, 2013 and 2014 and followed up to 2015. (2016). at <https://www.ons.gov.uk/peoplepopulationandcommunity/healthandsocialcare/conditionsand diseases/bulletins/cancersurvivalbystageatdiagnosisforenglandexperimentalstatistics/adultsdi agnosed20122013and2014andfollowedupto2015>

131. Gormally, E. et al. TP53 and KRAS2 Mutations in Plasma DNA of Healthy Subjects and Subsequent Cancer Occurrence: A Prospective Study. Cancer Res. 66, 6871-6876 (2006).

132. Amant, F. et al. Presymptomatic Identification of Cancers in Pregnant Women During Noninvasive Prenatal Testing. JAMA Oncol. 1, 814 (2015).

133. Bianchi, D. W. et al. Noninvasive Prenatal Testing and Incidental Detection of Occult Maternal Malignancies. JAMA 314, 162 (2015).

134. Beaver, J. A. et al. Detection of cancer DNA in plasma of patients with early-stage breast cancer. Clin. Cancer Res. 20, 2643-50 (2014).

135. Cohen, P. A. et al. Abnormal plasma DNA profiles in early ovarian cancer using a noninvasive prenatal testing platform: implications for cancer screening. BMC Med. 14, 126 (2016).

136. Gorges, T. M. et al. Enumeration and Molecular Characterization of Tumor Cells in Lung Cancer Patients Using a Novel In Vivo Device for Capturing Circulating Tumor Cells. Clin. Cancer Res. 22, 2197-206 (2016). 
137. Kinde, I. et al. Evaluation of DNA from the Papanicolaou test to detect ovarian and endometrial cancers. Sci. Transl. Med. 5, 167 ra4 (2013).

138. Nair, N. et al. Genomic Analysis of Uterine Lavage Fluid Detects Early Endometrial Cancers and Reveals a Prevalent Landscape of Driver Mutations in Women without Histopathologic Evidence of Cancer: A Prospective Cross-Sectional Study. PLOS Med. 13, e1002206 (2016).

139. Ross-Innes, C. S. et al. Risk stratification of Barrett's oesophagus using a non-endoscopic sampling method coupled with a biomarker panel: a cohort study. Lancet Gastroenterol. Hepatol. 2, 23-31 (2016).

140. Chan, K. C. A. et al. Early detection of nasopharyngeal carcinoma by plasma Epstein-Barr virus DNA analysis in a surveillance program. Cancer 119, 1838-1844 (2013).

141. Guerrero-Preston, R. et al. Molecular Triage of Premalignant Lesions in Liquid-Based Cervical Cytology and Circulating Cell-Free DNA from Urine, Using a Panel of Methylated Human Papilloma Virus and Host Genes. Cancer Prev. Res. 9, 915-924 (2016).

142. Martincorena, I. et al. High burden and pervasive positive selection of somatic mutations in normal human skin. Science 348, 880-886 (2015).

143. Genovese, G. et al. Clonal hematopoiesis and blood-cancer risk inferred from blood DNA sequence. N. Engl. J. Med. 371, 2477-87 (2014).

144. Fernandez-Cuesta, L. et al. Identification of Circulating Tumor DNA for the Early Detection of Small-cell Lung Cancer. EBioMedicine 10, 6-12 (2016).

145. Gormally, E., Caboux, E., Vineis, P. \& Hainaut, P. Circulating free DNA in plasma or serum as biomarker of carcinogenesis: practical aspects and biological significance. Mutat. Res. 635, 105-17 (2007).

146. Rosenfeld, N. et al. MicroRNAs accurately identify cancer tissue origin. Nat. Biotechnol. 26, 462-469 (2008).

147. Olsen, J. A. et al. A Minimally-invasive Blood-derived Biomarker of Oligodendrocyte Cell-loss in Multiple Sclerosis. EBioMedicine 10, 227-235 (2016).

148. Gerlinger, M. et al. Intratumor Heterogeneity and Branched Evolution Revealed by Multiregion Sequencing. N. Engl. J. Med. 366, 883-892 (2012).

149. Yates, L. R. et al. Subclonal diversification of primary breast cancer revealed by multiregion sequencing. Nat. Med. 21, 751-9 (2015).

150. Turajlic, S. \& Swanton, C. Metastasis as an evolutionary process. Science 352, 169-175 (2016).

151. Piotrowska, Z. et al. Heterogeneity Underlies the Emergence of EGFRT790 Wild-Type Clones Following Treatment of T790M-Positive Cancers with a Third-Generation EGFR Inhibitor. Cancer Discov. 5, 37-54 (2015).

152. De Mattos-Arruda, L. et al. Establishing the origin of metastatic deposits in the setting of multiple primary malignancies: the role of massively parallel sequencing. Mol. Oncol. 8, 150158 (2014).

153. Chan, K. C. A. et al. Noninvasive detection of cancer-associated genome-wide hypomethylation and copy number aberrations by plasma DNA bisulfite sequencing. Proc. Natl. Acad. Sci. 110, 18761-8 (2013).

154. Kuo, Y. Bin, Chen, J. S., Fand, C. W., Li, Y. S. \& Chan, E. C. Comparison of KRAS mutation analysis of primary tumors and matched circulating cell-free DNA in plasmas of patients with colorectal cancer. Clin. Chim. Acta 433, 284-289 (2014).

155. de Bruin, E. C. et al. Spatial and temporal diversity in genomic instability processes defines lung cancer evolution. Science 346, 251-256 (2014).

156. Zhang, J. et al. Intratumor heterogeneity in localized lung adenocarcinomas delineated by multiregion sequencing. Science 346, 256-9 (2014).

157. Oxnard, G. R. et al. Association Between Plasma Genotyping and Outcomes of Treatment With Osimertinib (AZD9291) in Advanced Non-Small-Cell Lung Cancer. J. Clin. Oncol. 34, 3375-3382 (2016).

158. Siravegna, G. et al. Clonal evolution and resistance to EGFR blockade in the blood of colorectal cancer patients. Nat. Med. 21, 795-801 (2015).

159. Tie, J. et al. Circulating Tumor DNA as an Early Marker of Therapeutic Response in Patients with Metastatic Colorectal Cancer. Ann. Oncol. 26, 1715-22 (2015).

160. Chabon, J. J. et al. Circulating tumour DNA profiling reveals heterogeneity of EGFR inhibitor resistance mechanisms in lung cancer patients. Nat. Commun. 7, 11815 (2016).

161. Karachaliou, N. et al. Association of EGFR L858R Mutation in Circulating Free DNA With Survival in the EURTAC Trial. JAMA Oncol. 1, 149-157 (2015).

162. Reck, M. et al. Circulating Free Tumor-derived DNA (ctDNA) Determination of EGFR Mutation Status in European and Japanese Patients with Advanced NSCLC: the ASSESS Study. J. Thorac. Oncol. 11, 1682-1689 (2016).

163. Han, B. et al. Determining the prevalence of EGFR mutations in Asian and Russian patients (pts) with advanced non-small-cell lung cancer (aNSCLC) of adenocarcinoma (ADC) and 
non-ADC histology: IGNITE study. Ann. Oncol. 26, i29-i44 (2015).

164. Douillard, J.-Y. et al. Gefitinib Treatment in EGFR Mutated Caucasian NSCLC. J. Thorac. Oncol. 9, 1345-1353 (2014).

165. Douillard, J.-Y. et al. First-line gefitinib in Caucasian EGFR mutation-positive NSCLC patients: a phase-IV, open-label, single-arm study. Br. J. Cancer 110, 55-62 (2014).

166. QIAGEN. therascreen EGFR Plasma RGQ PCR Kit Handbook - Version 2. (2016).

167. Mok, T. S. et al. Osimertinib or Platinum-Pemetrexed in EGFR T790M-Positive Lung Cancer. N. Engl. J. Med. NEJMoa1612674 (2016). doi:10.1056/NEJMoa1612674

168. U.S. Food and Drug Administration. Premarket Approval P150044 - Cobas EGFR MUTATION TEST V2. (2016). at $<$ ttp://www.accessdata.fda.gov/scripts/cdrh/cfdocs/cfpma/pma.cfm?id=P150044>

169. Thress, K. S. et al. EGFR mutation detection in ctDNA from NSCLC patient plasma: A crossplatform comparison of leading technologies to support the clinical development of AZD9291. Lung Cancer 90, 509-515 (2015).

170. Sorensen, B. S. et al. Monitoring of epidermal growth factor receptor tyrosine kinase inhibitorsensitizing and resistance mutations in the plasma DNA of patients with advanced non-small cell lung cancer during treatment with erlotinib. Cancer 120, 3896-3901 (2014).

171. Oxnard, G. R. et al. Noninvasive detection of response and resistance in EGFR-mutant lung cancer using quantitative next-generation genotyping of cell-free plasma DNA. Clin. Cancer Res. 20, 1698-705 (2014).

172. Remon, J. et al. Osimertinib benefit in EGFR-mutant NSCLC patients with T790M-mutation detected by circulating tumour DNA. Ann. Oncol. Off. J. Eur. Soc. Med. Oncol. (2017). doi:10.1093/annonc/mdx017

173. European Medicines Agency. Iressa: Public Assessment Report - Product Information. (2016). at <http://www.ema.europa.eu/docs/en_GB/document_library/EPAR_Product Information/human/001016/WC500036358.pdf>

174. European Medicines Agency. Tagrisso: Public Assessment Report - Product Information. (2016). at <http://www.ema.europa.eu/docs/en_GB/document_library/EPAR_Product_Information/human/004124/WC500202022.pdf>

175. US National Library of Medicine. ClinicalTrials.gov, https://clinicaltrials.gov/ct2/show/NCT02693535. (2017). at <https://clinicaltrials.gov/ct2/show/NCT02693535>

176. Schwaederle, M. et al. Use of Liquid Biopsies in Clinical Oncology: Pilot Experience in 168 Patients. Clin. Cancer Res. (2016). doi:10.1158/1078-0432.CCR-16-0318

177. US National Library of Medicine. ClinicalTrials.gov, https://clinicaltrials.gov/ct2/show/NCT02140463. (2015).

178. Remon, J. et al. Liquid biopsies for molecular profiling of mutations in non-small cell lung cancer (NSCLC) patients lacking tissue samples. in Molecular Analysis for Personalised Therapy (2016).

179. Institut Gustave Roussy. The Prospective MOSCATO 01 Trial Demonstrates that Molecular 'Portraits' Improve Outcome of Patients with Metastatic Cancer. (2016). at $<$ http://www.prnewswire.com/news-releases/the-prospective-moscato-01-trial-demonstratesthat-molecular-portraits-improve-outcome-of-patients-with-metastatic-cancer594507371.html>

180. Pishvaian, M. J. et al. A pilot study evaluating concordance between blood-based and patient-matched tumor molecular testing within pancreatic cancer patients participating in the Know Your Tumor (KYT) initiative. Oncotarget 5, (2016).

181. Heitzer, E. et al. Establishment of tumor-specific copy number alterations from plasma DNA of patients with cancer. Int. J. Cancer 133, 346-56 (2013).

182. Heitzer, E. Heitzer 2013 Plasma-seq supplementary methods. (2013).

183. Mohan, S. et al. Changes in Colorectal Carcinoma Genomes under Anti-EGFR Therapy Identified by Whole-Genome Plasma DNA Sequencing. PLoS Genet. 10, e1004271 (2014).

184. Kinde, I., Papadopoulos, N., Kinzler, K. W. \& Vogelstein, B. FAST-SeqS: A simple and efficient method for the detection of aneuploidy by massively parallel sequencing. PLoS One 7, e41162 (2012).

185. Abdueva, D. et al. Abstract 11541: Detection, frequency and actionability of recurrent copy number gains detected by non-invasive liquid biopsy of 3,942 lung and breast cancer samples. J. Clin. Oncol. 34, (2016).

186. Jiang, $P$. et al. Lengthening and shortening of plasma DNA in hepatocellular carcinoma patients. Proc. Natl. Acad. Sci. 112, E1317-25 (2015).

187. Romanel, A. et al. Plasma AR and abiraterone-resistant prostate cancer. Sci. Transl. Med. 7, 312re10-312re10 (2015).

188. Paweletz, C. P. et al. Bias-corrected targeted next-generation sequencing for rapid, multiplexed detection of actionable alterations in cell-free DNA from advanced lung cancer 
patients. Clin. Cancer Res. 22, 915-922 (2016).

189. Schreuer, M. et al. Quantitative assessment of BRAF V600 mutant circulating cell-free tumor DNA as a tool for therapeutic monitoring in metastatic melanoma patients treated with BRAF/MEK inhibitors. J. Transl. Med. 14, 95 (2016).

190. Marchetti, A. et al. Early Prediction of Response to Tyrosine Kinase Inhibitors by Quantification of EGFR Mutations in Plasma of NSCLC Patients. J. Thorac. Oncol. 10, 143743 (2015).

191. Xi, L. et al. Circulating Tumor DNA as an Early Indicator of Response to T-Cell Transfer Immunotherapy in Metastatic Melanoma. Clin. Cancer Res. 22, 5480-5486 (2016).

192. Xin, Y. et al. Patent CN105087789A - Method for detecting BCR and TCR immune repertoire in blood plasma cfDNA. (2015).

193. Tie, J. et al. Circulating tumor DNA analysis detects minimal residual disease and predicts recurrence in patients with stage II colon cancer. Sci. Transl. Med. 8, 346ra92-346ra92 (2016).

194. Garcia-Murillas, I. et al. Mutation tracking in circulating tumor DNA predicts relapse in early breast cancer. Sci. Transl. Med. 7, 302ra133-302ra133 (2015).

195. Reinert, T. et al. Analysis of circulating tumour DNA to monitor disease burden following colorectal cancer surgery. Gut 65, 625-34 (2015).

196. Olsson, E. et al. Serial monitoring of circulating tumor DNA in patients with primary breast cancer for detection of occult metastatic disease. EMBO Mol. Med. 7, 1034-1047 (2015).

197. Leary, R. J. et al. Development of personalized tumor biomarkers using massively parallel sequencing. Sci. Transl. Med. 2, 20ra14 (2010).

198. Mcbride, D. J. et al. Use of Cancer-Specific Genomic Rearrangements to Quantify Disease Burden in Plasma from Patients with Solid Tumors. Genes Chromosom. Cancer 49, 10621069 (2011).

199. Russo, M. et al. Acquired resistance to the TRK inhibitor entrectinib in colorectal cancer. Cancer Discov. 6, 36-44 (2016).

200. Misale, S. et al. Emergence of KRAS mutations and acquired resistance to anti EGFR therapy in colorectal cancer. Nature 486, 532-536 (2012).

201. Diaz, L. A. J. et al. The molecular evolution of acquired resistance to targeted EGFR blockade in colorectal cancers. Nature 486, 537-540 (2012).

202. Ilié, M. \& Hofman, P. Pros: Can tissue biopsy be replaced by liquid biopsy? Transl. Lung Cancer Res. 5, 420-423 (2016).

203. Thress, K. S. et al. Acquired EGFR C797S mutation mediates resistance to AZD9291 in nonsmall cell lung cancer harboring EGFR T790M. Nat. Med. 21, 560-562 (2015).

204. Russo, M. et al. Tumor heterogeneity and lesion-specific response to targeted therapy in colorectal cancer. Cancer Discov. 6, 147-53 (2015).

205. Morelli, M. P. et al. Characterizing the patterns of clonal selection in circulating tumor DNA from patients with colorectal cancer refractory to anti-EGFR treatment. Ann. Oncol. 26, 731736 (2015).

206. Gremel, G. et al. Distinct sub-clonal tumour responses to therapy revealed by circulating cellfree DNA. Ann. Oncol. 27, 1959-1965 (2016).

207. Ulz, P. et al. Whole-genome plasma sequencing reveals focal amplifications as a driving force in metastatic prostate cancer. Nat. Commun. 7, 12008 (2016).

208. European Medicines Agency. Guideline on good pharmacogenomic practice. (2016).

209. US National Library of Medicine. ClinicalTrials.gov, https://clinicaltrials.gov/ct2/show/NCT02284633. (2015).

210. US National Library of Medicine. ClinicalTrials.gov, https://clinicaltrials.gov/ct2/show/NCT02743910. (2016).

211. The Institute of Cancer Research Clinical Trials \& Statistics Unit. plasmaMATCH: A clinical trial aiming to assess the safety and activity of targeted treatments in patients with advanced breast cancer where the targetable mutation is identified through circulating tumour DNA screening. (2016). doi:10.1186/ISRCTN16945804

212. Naik, S. H., Schumacher, T. N. \& Perie, L. Cellular barcoding: A technical appraisal. Exp. Hematol. 42, 598-608 (2014).

213. El Messaoudi, S. et al. Circulating DNA as a Strong Multimarker Prognostic Tool for Metastatic Colorectal Cancer Patient Management Care. Clin. Cancer Res. 22, 3067-3077 (2016).

214. Spindler, K. L. G., Pallisgaard, N., Andersen, R. F. \& Jakobsen, A. Changes in mutational status during third-line treatment for metastatic colorectal cancer - Results of consecutive measurement of cell free DNA, KRAS and BRAF in the plasma. Int. J. Cancer 135, 2215$2222(2014)$.

215. Schwarzenbach, H., Hoon, D. S. B. \& Pantel, K. Cell-free nucleic acids as biomarkers in cancer patients. Nat. Rev. Cancer 11, 426-437 (2011). 
216. Sullivan, R. J. et al. Plasma-Based Monitoring of BRAF Mutations During Therapy for Malignant Melanoma Using Combined Exosomal RNA and Cell-Free DNA Analysis. J. Clin. Oncol. 33, 9017 (2015).

217. Ulz, P. et al. Inferring expressed genes by whole-genome sequencing of plasma DNA. Nat. Genet. 48, 1273-1278 (2016).

218. Shao, $\mathrm{H}$. et al. Chip-based analysis of exosomal mRNA mediating drug resistance in glioblastoma. Nat. Commun. 6, 6999 (2015).

219. Alix-Panabières, C. \& Pantel, K. Clinical Applications of Circulating Tumor Cells and Circulating Tumor DNA as Liquid Biopsy. Cancer Discov. 6, 479-491 (2016).

220. Best, M. G. et al. RNA-Seq of Tumor-Educated Platelets Enables Blood-Based Pan-Cancer, Multiclass, and Molecular Pathway Cancer Diagnostics. Cancer Cell 28, 666-676 (2015).

221. Reckamp, K. L. et al. A highly sensitive and quantitative test platform for detection of NSCLC EGFR mutations in urine and plasma. J. Thorac. Oncol. 11, 1690-1700 (2016).

222. Webb, S. The cancer bloodhounds. Nat. Biotechnol. 34, 1090-1094 (2016).

223. Long-Mira, E., Washetine, K. \& Hofman, P. Sense and nonsense in the process of accreditation of a pathology laboratory. Virchows Arch. 468, 43-49 (2016).

224. Melchior, L. et al. Multi-center evaluation of the novel fully-automated PCR-based Idylla BRAF Mutation Test on formalin-fixed paraffin-embedded tissue of malignant melanoma. Exp. Mol. Pathol. 99, 485-491 (2015).

225. Janku, F. et al. BRAF Mutation Testing in Cell-Free DNA from the Plasma of Patients with Advanced Cancers Using a Rapid, Automated Molecular Diagnostics System. Mol. Cancer Ther. 15, 1397-404 (2016).

226. Norris, A. L., Workman, R. E., Fan, Y., Eshleman, J. R. \& Timp, W. Nanopore sequencing detects structural variants in cancer. Cancer Biol. Ther. 17, 246-253 (2016).

227. Quick, J. et al. Real-time, portable genome sequencing for Ebola surveillance. Nature 530, 228-32 (2016).

228. Wei, S. \& Williams, Z. Rapid short-read sequencing and aneuploidy detection using minION nanopore technology. Genetics 202, 37-44 (2016).

229. Ip, C. L. C. et al. MinION Analysis and Reference Consortium: Phase 1 data release and analysis. F1000Research 4, 1075 (2015).

230. Loose, M., Malla, S. \& Stout, M. Real-time selective sequencing using nanopore technology. Nat Meth 13, 751-754 (2016).

231. Umetani, N., Hiramatsu, S. \& Hoon, D. S. B. Higher amount of free circulating DNA in serum than in plasma is not mainly caused by contaminated extraneous DNA during separation. Ann. N. Y. Acad. Sci. 1075, 299-307 (2006).

232. Willems, M., Moshage, H., Nevens, F., Fevery, J. \& Yap, S. H. Plasma collected from heparinized blood is not suitable for HCV-RNA detection by conventional RT-PCR assay. $J$. Virol. Methods 42, 127-130 (1993).

233. Jung, M., Klotzek, S., Lewandowski, M., Fleischhacker, M. \& Jung, K. Changes in Concentration of DNA in Serum and Plasma during Storage of Blood Samples. Clin. Chem. 49, 1028-1029 (2003).

234. Parpart-Li, S. et al. The effect of preservative and temperature on the analysis of circulating tumor DNA. Clin. Cancer Res. (2016). doi:10.1158/1078-0432.CCR-16-1691

235. El Messaoudi, S., Rolet, F., Mouliere, F. \& Thierry, A. R. Circulating cell free DNA: Preanalytical considerations. Clin. Chim. Acta 424, 222-230 (2013).

236. Sherwood, J. L. et al. Optimised pre-analytical methods improve KRAS mutation detection in circulating tumour DNA (ctDNA) from patients with non-small cell lung cancer (NSCLC). PLoS One 11, 1-14 (2016).

237. Toro, P. V. et al. Comparison of cell stabilizing blood collection tubes for circulating plasma tumor DNA. Clin. Biochem. 48, 993-998 (2015).

238. Kang, Q. et al. Comparative Analysis of Circulating Tumor DNA Stability In K3EDTA, Streck and CellSave Blood Collection Tubes. Clin. Biochem. 49, 1354-1360 (2016).

239. Medina Diaz, I. et al. Performance of Streck cfDNA blood collection tubes for liquid biopsy testing. PLoS One 11, e0166354 (2016).

240. Norton, S. E., Lechner, J. M., Williams, T. \& Fernando, M. R. A stabilizing reagent prevents cell-free DNA contamination by cellular DNA in plasma during blood sample storage and shipping as determined by digital PCR. Clin. Biochem. 46, 1561-1565 (2013).

241. Dietz, S. et al. Low Input Whole-Exome Sequencing to Determine the Representation of the Tumor Exome in Circulating DNA of Non-Small Cell Lung Cancer Patients. PLoS One 11, e0161012 (2016).

242. Schmitt, M. W. et al. Detection of ultra-rare mutations by next-generation sequencing. Proc. Natl. Acad. Sci. 109, 14508-14513 (2007).

243. Zonta, E., Garlan, F., Perez-Toralla, K. \& Taly, V. Multiplex Detection of Rare Mutations by Picoliter Droplet Based Digital PCR: Sensitivity and Specificity Considerations. PLoS One 11, 
e0159094 (2016).

244. Marziali, A., Pel, J., Bizzotto, D. \& Whitehead, L. A. Novel electrophoresis mechanism based on synchronous alternating drag perturbation. Electrophoresis 26, 82-90 (2005).

245. Thompson, J. D., Shibahara, G., Rajan, S., Pel, J. \& Marziali, A. Winnowing DNA for rare sequences: Highly specific sequence and methylation based enrichment. PLoS One 7, e31597 (2012).

246. Kidess, E. et al. Mutation profiling of tumor DNA from plasma and tumor tissue of colorectal cancer patients with a novel, high-sensitivity multiplexed mutation detection platform. Oncotarget 6, 2549-61 (2015).

\section{Highlighted References}

Diehl, F. et al. Circulating mutant DNA to assess tumor dynamics. Nat. Med. 14, 985990 (2008).

Demonstration that ctDNA dynamics in patients undergoing treatment for colorectal cancer reflect tumour responses and progression, and that ctDNA detection after surgery could detect residual disease as a prognostic factor.

Forshew, T. et al. Noninvasive Identification and Monitoring of Cancer Mutations by Targeted Deep Sequencing of Plasma DNA. Sci. Transl. Med. 4, 136ra68-136ra68 (2012).

Identification of cancer mutations directly in plasma using a next-generation sequencingbased assay, and application of this method to quantify and monitor multiple mutations.

Murtaza, M. et al. Non-invasive analysis of acquired resistance to cancer therapy by sequencing of plasma DNA. Nature 497, 108-12 (2013).

Exome sequencing of cfDNA from serial plasma samples to study clonal evolution and to track ctDNA dynamics in high-burden disease.

Sun, K. et al. Plasma DNA tissue mapping by genome-wide methylation sequencing for noninvasive prenatal, cancer, and transplantation assessments. Proc. Natl. Acad. Sci. 201508736 (2015). doi:10.1073/pnas.1508736112.

Analysis of the relative contributions of different tissues to the total cfDNA pool using methylation deconvolution. 
Mouliere, F. et al. High Fragmentation Characterizes Tumour-Derived Circulating DNA. PLoS One 6, e23418 (2011).

Specific characterisation of ctDNA fragmentation patterns and biological properties using an animal model system and clinical samples.

Bettegowda, C. et al. Detection of circulating tumor DNA in early- and late-stage human malignancies. Sci. Transl. Med. 6, 224ra24 (2014).

A comprehensive quantitative analysis found varying levels of ctDNA across patients with distinct cancer types, and provided indicative ranges of ctDNA levels across the stages of disease.

Newman, A. M. et al. Integrated digital error suppression for improved detection of circulating tumor DNA. Nat Biotechnol 34, 547-55 (2016).

An error suppression method for capture-enrichment sequencing which uses a combination of in silico elimination of stereotyped background artifacts, and molecular barcoding.

Garcia-Murillas, I. et al. Mutation tracking in circulating tumor DNA predicts relapse in early breast cancer. Sci. Transl. Med. 7, 302ra133-302ra133 (2015).

Demonstration of the use of ctDNA for detection of MRD in early-stage breast cancer following treatment with curative intent.

Thierry, A. R. et al. Clinical validation of the detection of KRAS and BRAF mutations from circulating tumor DNA. Nat. Med. 20, 430-435 (2014).

A blinded prospective multi-centre study comparing KRAS and BRAF mutation status in tumour and plasma using an allele-specific quantitative PCR approach.

Leary, R. J. et al. Detection of Chromosomal Alterations in the Circulation of Cancer Patients with Whole-Genome Sequencing. Sci. Transl. Med. 4, 1-21 (2012).

WGS of cfDNA from plasma identified copy number alterations and rearrangements. 
Tie, J. et al. Circulating tumor DNA analysis detects minimal residual disease and predicts recurrence in patients with stage II colon cancer. Sci. Transl. Med. 8, (2016).

A prospective cohort study of 230 patients with resected stage II colon cancer demonstrated that post-operative ctDNA detection predicts a poorer recurrence-free survival.

Heitzer, E. et al. Tumor-associated copy number changes in the circulation of patients with prostate cancer identified through whole-genome sequencing. Genome Med. 5, 30 (2013).

A demonstration that WGS of plasma cfDNA at a shallow sequencing depth can establish copy number profiles in patients with prostate cancer with a rapid turnaround time.

Misale, S. et al. Emergence of KRAS mutations and acquired resistance to anti EGFR therapy in colorectal cancer. Nature 486, 532-536 (2012).

Serial ctDNA analysis identified KRAS mutant allels in the plasma of cetuximab-treated patients 10 months before disease relapse was identified by imaging.

Thress, K. S. et al. Acquired EGFR C797S mutation mediates resistance to AZD9291 in non-small cell lung cancer harboring EGFR T790M. Nat. Med. 21, 560-562 (2015).

Serial ctDNA monitoring of patients with lung cancer treated with AZD9291, an EGFR inhibitor, revealed a diversity of resistance mechanisms to therapy.

Jiang, P. et al. Lengthening and shortening of plasma DNA in hepatocellular carcinoma patients. Proc. Natl. Acad. Sci. 112, E1317-25 (2015).

Genome-wide analysis of plasma DNA from patients with hepatocellular carcinoma found that short molecules of cfDNA were enriched for tumour-associated copy number alterations.

Oxnard, G. R. et al. Association Between Plasma Genotyping and Outcomes of Treatment With Osimertinib (AZD9291) in Advanced Non-Small-Cell Lung Cancer. J. Clin. Oncol. 34, 3375-3382 (2016).

In retrospective analysis, patients with NSCLC who were positive for $E G F R^{T 790 \mathrm{M}}$ in plasma 
showed outcomes with osimertinib that were equivalent to patients positive by a tissue-based assay, supporting the use of plasma analysis to avoid tumour biopsies for patients who are positive for T790M in plasma.

Chabon, J. J. et al. Circulating tumour DNA profiling reveals heterogeneity of EGFR inhibitor resistance mechanisms in lung cancer patients. Nat. Commun. 7, 11815 (2016).

ctDNA analysis by a large panel of genes was used to identify multiple hetereogeneous resistance mechanisms in patients with NSCLC treated by rociletinib, an EGFR-inhibitor.

\section{Glossary}

Liquid biopsy - analysis of tumour material (e.g. cells or nucleic acids) obtained in a minimally or non-invasive manner, through sampling of blood or other body fluids.

Hybrid-capture sequencing - DNA sequencing of kilobases to megabases of the genome, in which DNA to be sequenced is selected using complementary oligonucleotide baits that hybridise to the target DNA. The DNA is then captured in solution, commonly through binding to magnetic beads.

Limit of detection - The threshold below which mutations cannot be confidently discriminated from background noise; for sequencing-based approaches, this is often determined by technical artefacts such as PCR or sequencing errors.

Stem mutations - Mutations that occur early in a cancer's development and are present in all cells.

Private mutations - Mutations that are present only in a specific region of a tumour, or in a subset of cells, due to intratumour heterogeneity.

Molecular barcoding - Unique molecular sequences that are added to each molecule when creating a sequencing library, so that reads originating from the same molecule may be identified and the consensus taken, correcting for some PCR or sequencing errors. 
Mutant allele fraction - the proportion of mutant DNA fragments at a given locus.

Mutant allele concentration - the number of mutant DNA fragments at a given locus, per unit volume.

Digital PCR - Many micro-litre or nano-litre scale PCR reactions are run in parallel within physically separated reaction chambers, or droplets in an emulsion (droplet dPCR, ddPCR). By partitioning molecules into hundreds or up to millions of reactions, rare mutant molecules may be accurately identified and quantified.

Targeted Sequencing - massively parallel (next generation) sequencing that uses methods such as PCR amplification or hybrid capture to focus on a subset of the genome, which can range from few genes or mutation loci, to large fractions of the genome such as the entire exome. Smaller panels yield higher sequencing depth at lower costs.

\section{Acknowledgements}

The authors would like to thank all members of the Rosenfeld Lab, in particular Christopher G Smith, Davina Gale, Keval M Patel and Wendy N Cooper for expert advice and proofreading of this manuscript. We would like to thank Dennis Lo for providing the image used in Figure 3a. We would like to acknowledge the support of The University of Cambridge, Cancer Research UK (grant numbers A11906, A20240, A15601) (to N.R., J.D.B.), the European Research Council under the European Union's Seventh Framework Programme (FP/20072013) / ERC Grant Agreement n. 337905 (to N.R.), and Hutchison Whampoa Limited (to N.R.), AstraZeneca (to R.B., S.P.), the Cambridge Experimental Cancer Medicine Centre (ECMC) (to R.B., S.P.), and NIHR Biomedical Research Centre (BRC) (to R.B., S.P.). J.G.C. acknowledges clinical fellowship support from SEOM.

\section{Competing interests statement}

NR is the CSO of Inivata. NR and JDB are cofounders and shareholders of Inivata. NR and FM are co-inventors of patent applications describing methods for analysis of DNA fragments and applications of ctDNA. 